Please do not remove this page

RMIT

UNIVERSITY

\title{
Dynamics of a model colloidal suspension from dilute to freezing
}

Hannam, Stephen; Daivis, Peter; Bryant, Gary

https://researchrepository.rmit.edu.au/esploro/outputs/9921860115801341/filesAndLinks?institution=61RMIT_INST\&index=null

Hannam, S., Daivis, P., \& Bryant, G. (2016). Dynamics of a model colloidal suspension from dilute to freezing. Physical Review E - Statistical, Nonlinear, and Soft Matter Physics, 94(1), 1-15.

https://doi.org/10.1103/PhysRevE.94.012619

Document Version: Accepted Manuscript

Published Version: https://doi.org/10.1103/PhysRevE.94.012619

Repository homepage: https://researchrepository.rmit.edu.au

(c) 2016 American Physical Society

Downloaded On 2023/04/26 21:17:23 +1000

Please do not remove this page 
Thank you for downloading this document from the RMIT Research Repository.

The RMIT Research Repository is an open access database showcasing the research outputs of RMIT University researchers.

RMIT Research Repository: http://researchbank.rmit.edu.aul

\author{
Citation: \\ Hannam, S, Daivis, P and Bryant, G 2016, 'Dynamics of a model colloidal \\ suspension from dilute to freezing', Physical Review E - Statistical, Nonlinear, and \\ Soft Matter Physics, vol. 94, no. 1, 12619, pp. 1-15.
}

See this record in the RMIT Research Repository at:

https://researchbank.rmit.edu.au/view/rmit:38559

Version: Accepted Manuscript

Copyright Statement:

(C) 2016 American Physical Society

Link to Published Version:

http://dx.doi.org/10.1103/PhysRevE.94.012619 
Please cite as:

Hannam, S.D.W., Daivis, P.J., Bryant, G.

"Dynamics of a model colloidal suspension from dilute to freezing," Physical Review E - Statistical, Nonlinear, and Soft Matter Physics 94, 012619 (2016). 


\title{
Dynamics of a model colloidal suspension from dilute to freezing
}

\author{
S. D. W. Hannam, P. J. Daivis, ${ }^{*}$ and G. Bryant \\ School of Science and Centre for Molecular and Nanoscale Physics, \\ RMIT University, GPO Box 2476, Melbourne Vic 3001, Australia.
}

(Dated: July 10, 2016)

\begin{abstract}
Molecular dynamics simulation was used to study a model colloidal suspension at a range of packing fractions from the dilute limit up to the freezing point. This study builds on previous work by the authors which modelled the colloidal particles with a hard-core surrounded by a Weeks-ChandlerAnderson potential with modified interaction parameters, and included an explicit solvent. In this work we study dynamical properties of the model by first calculating the velocity autocorrelation function, the self diffusion coefficient and the mutual diffusion coefficient. We also perform detailed calculations of the colloidal particle intermediate scattering function to study the change in dynamics leading up to the freezing point, and to determine whether the current model can be used to interpret light scattering experiments. We then perform a multiexponential analysis on the intermediate scattering function results and find that the data is fitted well by the sum of two exponentials, which is in line with previous analysis of experimental colloidal suspensions. The amplitudes and decay coefficients of the two modes are determined over a large range of wavevectors at packing fractions leading up to the freezing point. We found that the minimum wavevector needed to be effectively in the macroscopic diffusive limit decreased as the packing fraction increased, and a simple extrapolation shows the minimum wavevector going to zero at the melting point. Lastly, the ratio of the two decay coefficients is compared to the scaling law proposed by Segrè and Pusey [Phys. Rev. Lett. 77, 771 (1996)]. It was found that the ratio was not constant, but instead was wavevector dependent.
\end{abstract}

\section{INTRODUCTION}

Colloidal systems are ideal for studies of solidification mechanisms. An understanding of crystallization processes in colloidal systems aids in the understanding of phase transitions in other soft matter systems [1, 2], with application to materials design [3] and to biology [4]. Crystallization in colloidal suspensions can be studied via light scattering experiments, often using dynamic light scattering (DLS) or X-ray photon correlation spectroscopy (XPCS) [5].

Typical model systems used in light scattering experiments consist of suspensions of spherical particles which are stabilized against aggregation by coating the surface with a short-chained polymer (steric stabilization) or with a charged ionic layer (charge stabilization). The computational model used in this work does not include electrostatic interactions. Therefore, it aims to replicate the dynamics of sterically stabilized suspensions where the interaction is steeply repulsive and is often modeled with hard-sphere interactions. The pioneering work using DLS to study the dynamics of dense colloidal suspensions was done by Pusey, van Megen and collaborators [6-11].

Computational models are a useful complement to experimental investigations. But although the computer power available is ever increasing, a full molecular dynamics (MD) treatment of all the interactions present in the system is still computationally unattainable. Because of the complexity of experimental systems, most simulations resort to idealized descriptions, often model-

* Electronic mail: peter.daivis@rmit.edu.au ing the systems as single component hard-spheres using event driven MD [12] which completely ignores the presence of the solvent. This means the particles in these models move with ballistic dynamics, rather than diffusing through a solvent. These models neglect effects such as the viscoelasticity of the solvent and the momentum transfer that occurs via the solvent [13].

To implicitly take into account the solvent, Brownian dynamics (BD) treats the fluid as a continuum represented by frictional and random forces. This introduces an effective drag on the hard-spheres, but usually does not include multi body hydrodynamic interactions (HIs) from the solvent [14, 15]. Incorporating 2-body $\mathrm{HIs}$ in $\mathrm{BD}$ in a simplified way requires the use of hydrodynamic tensors such as the Yamakawa-Rotne-Prager (YRP) tensors [16-18]. However, BD-YRP hydrodynamics is only valid for relatively dilute suspensions and can be quite computationally expensive. Alternative techniques that have been developed to include HIs include Lattice-Boltzmann [19, 20], Dissipative Particle Dynamics [21], and Stochastic Rotation Dynamics [22, 23], all of which involve coarse-graining the solvent.

Few attempts have been made to include solvent explicitly into the simulation by calculating the equations of motion for both the colloidal particles and the solvent directly. This is simply because in order to match the size and mass ratio of experimental colloidal suspensions, the simulation would require in the order of tens of millions of solvent molecules for every colloidal particle. This is clearly beyond the reach of current computational capabilities, so smaller size and mass ratios have to be used. Vrabecz and Toth [24] studied the effect of explicitly adding a second smaller HS particle (1/5th and $1 / 10$ th the diameter of the larger particle) on the struc- 
tural properties of the fluid. They found that including the second smaller species caused a change in the radial distribution function of the larger particles in the fluid. This was evident through sharpening in the main peak, showing that the presence of the smaller particles causes a very strong depletion attraction between the larger particles.

Previous work done by the authors expanded on the explicit solvent model by including a second species with a smaller mass $(1 / 50$ th the mass of the larger particles) and smaller size $(1 / 4$ th the diameter) to represent the solvent [25]. In this model the colloidal particles were modeled using a Weeks-Chandler-Anderson (WCA) potential which was modified to include a hard-core, while the solvent was modeled using a simple WCA potential. In agreement with previous work, it was found that introduction of the second smaller species caused strong depletion attractions between the larger species [24]. These strong depletion forces are not present in experimental HS colloidal suspensions, and are purely due to the relatively small size ratio that had to be employed in the simulations due to computational limitations. Because the diameter of the two particles is of the same order, there is an excluded volume around the colloidal particles that gives rise to significant depletion effects.

Since the depletion effects were caused by the large excluded volume, it could be effectively eliminated by reducing the hard-core parameter in the colloidal-solvent pair interaction [25]. This allowed the solvent to effectively penetrate the colloid, which can be seen as unphysical, but the model was found to replicate the properties of an experimental colloidal suspension. The static structure factor, phase behavior and crystal structure were all found to agree with experimental results. With the apparent success of this model in replicating the behavior of real colloidal suspensions for these few key features, it remains to be seen how well it matches the dynamical behavior as represented by other properties (such as diffusion coefficients and the intermediate scattering function) and whether the model can be used as a complement to light scattering experiments.

Therefore, one of the aims of this work is to expand on the previous work by calculating a number of key transport coefficients over a wide range of colloid packing fractions (also referred to as volume fractions) from the dilute fluid up to the freezing point. Then, we compare the calculations of the model with available experimental results to determine the level of agreement. The other goal of this paper is to use this model to obtain accurate calculations for the intermediate scattering function at packing fractions approaching the freezing point.

In the liquid state, the empirical fit to the intermediate scattering function usually takes the form of a single or double exponential [26, 27]. In extremely dilute systems at low wavevector, DLS yields a single exponential decay with a wavevector independent diffusion coefficient. This is in line with what was found in previous work using this model [25]. As the packing fraction increases the diffu- sion coefficient becomes wavevector dependent, but the decay is still well approximated by a single exponential.

At moderate packing fractions, a second decay mode is observed and the data is fitted with two exponentials with different effective diffusion coefficients [9, 27]. These two empirical modes are often associated with short-time and long-time diffusion coefficients, where the former is associated with movement of the colloidal particles in its local cage while the latter is associated with diffusion over larger length scales [9]. This interpretation of the two modes has not been verified, and an exact relationship between the decay coefficients and transport or thermodynamic properties has not been made. Even so, Segrè and Pusey [28] proposed an empirical scaling law where the ratio of the two effective diffusion coefficients is approximately constant for highly concentrated colloidal suspensions over a broad range of wavevectors around the structure factor peak.

The validity of this scaling law has been called into question, with Lurio et al. [29] failing to observe the scaling in XPCS experiments on a charge-stabilised colloidal suspension. This was thought to be either because the colloids were charge stabilized (rather than sterically stabilized) or because XPCS gives different results to DLS. Martinez et al. [5] showed that the results of XPCS and DLS experiments are consistent, ruling out the latter explanation. They were able to see the scaling behavior over several decades in time but not in the long time limit. More recent work by Orsi et al. [27] studied a system very similar to that used by Martinez and Segrè, and found that the scaling law did hold for high concentration sterically stabilized colloids.

A systematic study of the individual decay modes over a large range of packing fractions and wavevectors is difficult to do experimentally. In particular it is difficult to access low wavevectors using existing techniques. Therefore, we choose to use MD to calculate the intermediate scattering function and use a multiexpontial analysis to decompose its individual contributions in order to complement the experimental investigation.

The outline of this paper is as follows: first we give a summary of the computational model used, and describe how we calculated the self and mutual diffusion coefficients in equilibrium MD using time correlation functions. Then, we systematically study the behavior of the correlation functions and the self and mutual diffusion coefficient from an extremely dilute state up to packing fractions just below the freezing point in order to observe any major changes that occur in the approach to freezing. Finally, a multiexponential analysis is carried out on the colloidal particle intermediate scattering function from small wavevectors to just past the structure factor peak. This is done for higher packing fractions approaching the freezing point. We discuss the individual decay modes that are observed and their wavevector and packing fraction dependence. We then test the scaling relationship proposed by Segrè and Pusey [28] to see if we also find a constant ratio of the short and long time 
diffusion coefficients.

\section{THEORY}

\section{A. Thermodynamic and transport coefficients}

Transport coefficients can be determined by calculating time correlation functions (TCFs) and using GreenKubo relations [30]. In general, a time correlation function $C(\mathbf{r}, \tau)$ often takes the form:

$$
C(\mathbf{r}, \tau)=\langle A(\mathbf{r}, \tau) A(\mathbf{r}, 0)\rangle
$$

where $A(\mathbf{r}, \tau)$ is a microscopic property which may depend on position $\mathbf{r}$ and delay time $\tau$. $\langle\ldots\rangle$ indicates ensemble averaging. In Fourier space Eq. (1) takes the form

$$
C(k, \tau)=\left\langle A(\mathbf{k}, \tau) A^{*}(\mathbf{k}, 0)\right\rangle
$$

where $A(\mathbf{k}, \tau)$ is now the spatial Fourier transform of the microscopic variable $A(\mathbf{r}, \tau)$ and ${ }^{*}$ indicates the complex conjugate. The wave vector $\mathbf{k}$ being studied in MD simulation must be consistent with the periodic boundary conditions of the simulation box:

$$
\mathbf{k}=\frac{2 \pi}{L}\left(n_{1}, n_{2}, n_{3}\right)
$$

where $n_{i}$ is an integer and $L$ is the length of the simulation box (in this work the box is cubic so $L_{x}=L_{y}=L_{z}$ ). The correlation function $C(k, \tau)$ only depends on the magnitude $k=|\mathbf{k}|$ as the average is done over all $\mathbf{k}$ of equal magnitude (as the fluid is isotropic). Also, from Eq. (3) we see that the lowest $k$ value that can be measured in an MD simulation is $k=2 \pi / L$.

In this work, we study correlation functions of particular microscopic variables that give useful information on properties of the colloidal suspension. The self diffusion coefficient of the colloidal particles $D_{s}$ can be calculated from the integral of the velocity autocorrelation function $C(\tau)$ by $[31]$ :

$$
\begin{aligned}
D_{s} & =\int_{0}^{\infty} C(\tau) d \tau \\
& =\frac{1}{3} \int_{0}^{\infty}\left\langle\mathbf{v}_{i}(\tau) \cdot \mathbf{v}_{i}(0)\right\rangle d \tau
\end{aligned}
$$

where $\mathbf{v}_{i}$ is the velocity of colloidal particle $i$ and the average is done over all colloidal particles. Self diffusion is related to the diffusion of a particle in the absence of temperature and/or concentration gradients. But, in the presence of such gradients other transport coefficients are defined. The linearized macroscopic diffusion equation for species 1 (which represents the colloidal particles) in a binary fluid is [32]:

$$
\frac{\partial c_{1}}{\partial t}=D_{m} \nabla^{2} c_{1}+D^{\prime} \nabla^{2} T
$$

where $c_{i}$ refers to the mass fraction of species $i, D_{m}$ is the mutual diffusion coefficient, $D^{\prime}$ is the thermaldiffusion coefficient and $T$ is the temperature. The coefficients $D_{m}$ and $D^{\prime}$ can be calculated from non-equilibrium MD simulation by setting up a system with a concentration/temperature gradient. They can also be calculated from equilibrium MD by relating them to the phenomenological coefficients $L_{\alpha \beta}$ which can be calculated from TCFs using Green-Kubo relations. As we will see later, the mutual diffusion coefficient $D_{m}$ governs the decay of the intermediate scattering functions in the low wavevector limit.

A well known expression for the mutual diffusion coefficient $D_{m}$ is given by [32]:

$$
D_{m}=\frac{L_{11}}{\rho c_{2} T}\left(\frac{\partial \mu_{1}}{\partial c_{1}}\right)_{p, T}
$$

where $\rho$ is the total mass density of the fluid and $\mu_{1}$ is the chemical potential per unit mass of the colloidal particles. $L_{11}$ is the phenomenological coefficient given in the Green-Kubo relations as

$$
L_{11}=\lim _{\tau \rightarrow \infty} \int_{0}^{\tau} A_{11}(\tau) d \tau
$$

and

$$
A_{11}(\tau)=\frac{V}{3 k_{B} T}\left\langle\mathbf{J}_{1}(\tau) \cdot \mathbf{J}_{1}(0)\right\rangle
$$

where $k_{B}$ is Boltzmann's constant and $V$ the volume of the system. The microscopic expression for the diffusive mass flux $\mathbf{J}_{1}$ of the colloidal particles takes the form

$$
\mathbf{J}_{1}=\frac{1}{V} \sum_{i=1}^{N_{1}} m_{1}\left(\mathbf{v}_{i}-\mathbf{v}\right)
$$

where $m_{1}$ is the mass of a colloidal particle and $\mathbf{v}$ is the average streaming velocity $(\mathbf{v}=0$ for an equilibrium fluid).

Apart from the usual transport coefficients we also calculated the intermediate scattering function $F_{\alpha \beta}(k, \tau)$ which is also measured in light scattering experiments. This can be done in equilibrium MD simulation by calculating the correlation function of the Fourier transform of the number density:

$$
n_{\alpha}(\mathbf{k}, t)=\frac{1}{\sqrt{N}} \sum_{i=1}^{N_{\alpha}} \exp \left(-\mathbf{k} \cdot \mathbf{r}_{i}(t)\right)
$$

where $N$ is the total number of particles, $N_{\alpha}$ is the number of particles of species $\alpha$ and $F_{\alpha \beta}(k, \tau)$ is given as

$$
F_{\alpha \beta}(k, \tau)=\frac{\left\langle n_{\alpha}(\mathbf{k}, \tau) n_{\beta}^{*}(\mathbf{k}, 0)\right\rangle}{S_{\alpha \beta}(k)}
$$

where the static structure factor $S_{\alpha \beta}(k)$ is the zero delay time value $F_{\alpha \beta}(k, \tau=0)$

$$
S_{\alpha \beta}(k)=\left\langle n_{\alpha}(\mathbf{k}, 0) n_{\beta}^{*}(\mathbf{k}, 0)\right\rangle .
$$


From the calculations of the $F_{\alpha \beta}(k, \tau)$ we are able to make comparisons with experimental data.

The decay of $F_{11}(k, \tau)$ can be related to the mutual diffusion coefficient $D_{m}$ in the macroscopic diffusive limit. From the thermodynamic point of view $D_{m}$ relates the diffusive mass flux to the gradient in the concentration. From the microscopic point of view such gradients arise in an equilibrium suspension from local fluctuations, and the decay of these fluctuations is governed by the same equation as the decay of macroscopic gradients.

Therefore, starting with the balance equation for the mass fraction of species 1 we can Fourier transform Eq. (5) into $k$-space which gives:

$$
\frac{\partial c_{1}(k, t)}{\partial t}=-D_{m} k^{2} c_{1}(k, t)
$$

where we have neglected thermal-diffusion as this effect is small in comparison to mutual diffusion. After multiplying both sides of Eq. (13) by the complex conjugate of the initial time value $c_{1}^{*}(k, 0)$ and ensemble averaging, the solution becomes

$$
F_{11}(k, \tau)=\frac{\left\langle c_{1}(k, \tau) c_{1}^{*}(k, 0)\right\rangle}{\left\langle\left|c_{1}(k, 0)\right|^{2}\right\rangle}=\exp \left(-k^{2} D_{m} \tau\right)
$$

which corresponds to the intermediate scattering function of the colloidal particles. This macroscopic relationship is only expected to hold in the macroscopic diffusive limit. Therefore, the mutual diffusion coefficient governs the decay of the intermediate scattering function in the $k \rightarrow 0$ limit.

\section{B. Calculation of the thermodynamic factor}

In order to calculate the mutual diffusion coefficient $D_{m}$ (defined in Eq. (6)), accurate values are needed for both the thermodynamic factor $\partial \mu_{1} / \partial c_{1}$ and phenomenological coefficient $L_{11}$. $L_{11}$ can be calculated from Green-Kubo theory, but $\partial \mu_{1} / \partial c_{1}$ is more difficult to calculate. One of the best ways to calculate it is using the theory presented by Kirkwood and Buff (KB) which relates the thermodynamic factor to the integral of the radial distribution functions $G_{\alpha \beta}$ [33]. A conversion of the original $\mathrm{KB}$ relations to the mass fraction units was done by Zhou and Miller [34] and is given for a binary fluid as

$$
\left(\frac{\partial \mu_{1}}{\partial c_{1}}\right)_{p, T}=\frac{V^{2}}{\rho\left\langle|c|^{2}\right\rangle}
$$

where

$$
\begin{aligned}
& \left\langle|c|^{2}\right\rangle= \\
& \frac{m_{1}^{2} m_{2}^{2} x_{1} x_{2} n^{2} N}{\rho^{4}}\left[1+x_{1} x_{2} n\left(G_{11}+G_{22}-2 G_{12}\right)\right]
\end{aligned}
$$

Here $m_{\alpha}$ and $x_{\alpha}$ are the mass and number fraction of species $\alpha$ respectively, and $n$ is the total number density of the fluid. The volume integrals of the radial distribution functions $G_{\alpha \beta}$ are calculated from

$$
G_{\alpha \beta}=\int\left(g_{\alpha \beta}(\boldsymbol{r})-1\right) d \mathbf{r}=4 \pi \int r^{2}\left(g_{\alpha \beta}(r)-1\right) d r
$$

where $g_{\alpha \beta}(r)$ is the radial distribution function of species $\alpha$ and $\beta$. These integrals can be difficult to calculate as statistical error in $g_{\alpha \beta}$ at large $r$ is magnified by the factor of $r^{2}$, so the numerical integrals may not converge. Therefore, calculation of $G_{\alpha \beta}$ usually requires very accurate data for $g_{\alpha \beta}(r)$ and fitting a function to the tail to evaluate the integral $[35,36]$.

Instead of calculating this integral, it can be simpler to calculate the values of $G_{\alpha \beta}$ through the static structure factors $S_{\alpha \beta}(k)$. It is well known that the partial structure factors are related to the Fourier transforms of the radial distribution functions through [31]

$$
S_{\alpha \beta}(k)=x_{\alpha} \delta_{\alpha \beta}+x_{\alpha} x_{\beta} n \int g_{\alpha \beta}(r) \exp (i \mathbf{k} \cdot \mathbf{r}) d \mathbf{r} .
$$

Assuming that the fluid is isotropic and writing the constant part of the radial distribution explicitly this can be rewritten as

$$
\begin{aligned}
S_{\alpha \beta}(k) & =x_{a} \delta_{\alpha \beta}+4 \pi x_{\alpha} x_{\beta} n \int r^{2}\left[g_{\alpha \beta}(r)-1\right] \exp (i \mathbf{k} \cdot \mathbf{r}) d r \\
& +(2 \pi)^{3} x_{\alpha} x_{\beta} n \delta(k)
\end{aligned}
$$

Comparing this with the expression given for $G_{\alpha \beta}$ in Eq. (17) we see that, if we ignore the contribution of the delta function at zero wave vector, we can write

$$
\begin{aligned}
G_{\alpha \beta} & =4 \pi \int r^{2}\left[g_{\alpha \beta}(r)-1\right] d r \\
& =\lim _{k \rightarrow 0} 4 \pi \int r^{2}\left[g_{\alpha \beta}(r)-1\right] \exp (i \mathbf{k} \cdot \mathbf{r}) d r
\end{aligned}
$$

therefore

$$
G_{\alpha \beta}=\frac{1}{x_{\alpha} x_{\beta} n}\left[\lim _{k \rightarrow 0} S_{\alpha \beta}(k)-x_{\alpha} \delta_{\alpha \beta}\right] .
$$

So by calculating the low- $k$ values of the partial structure factors $S_{\alpha \beta}(k)$, and extrapolating $k \rightarrow 0$, the values of $G_{\alpha \beta}$ can be calculated in a much simpler way. This method offers a much easier method of calculation $G_{\alpha \beta}$ than through the integral given in Eq. (17) directly. An alternate derivation of a relationship between the thermodynamic factors and the $S_{\alpha \beta}(k \rightarrow 0)$ values was previously done by Nichols and coworkers [37], though their definition of the thermodynamic factor differs.

\section{Multiexponential analysis}

As stated earlier, the intermediate scattering function is defined as the autocorrelation function of a Fourier 
component of the number density. Barocchi and coauthors [38-40] showed that the complete behavior of any normalized autocorrelation function of a classical manybody system can be described by a generalized Langevin equation, the exact solution of which can be written as an infinite sum of exponential functions

$$
C(t)=\sum_{j=1}^{\infty} A_{j} \exp \left(z_{j} t\right)
$$

where $A_{j}$ and $z_{j}$ are mode amplitudes and decay coefficients respectively. Such modes can be associated with relaxation channels in the system. If $A_{j}$ and $z_{j}$ are complex quantities, the corresponding mode and its complex conjugate are both present in the series and, taken together, they represent an exponentially damped oscillation. Otherwise, real $A_{j}$ and $z_{j}$ define a purely exponential decay.

An approximate solution to the generalized Langevin equation can be found by truncating Eq. (22) at a finite number of terms. The behavior of the coefficients can then be studied by fitting the resulting function to experimental/simulation data. This procedure can be difficult, due to the large number of free fitting parameters.

Barocchi and coauthors found that the number of free fitting parameters can be reduced by constraining the solution. They showed that the zero time properties of the solution given in Eq. (22) must obey the relation:

$$
\left(\frac{d^{m} C(t)}{d t^{m}}\right)_{t=0}=0
$$

where $m$ is an odd integer. When a finite number of exponential terms is retained, Eqs. (22) and (23) can only be valid for $m$ up to a certain value depending on the approximation level and the model assumed. The combination of Eqs. (22) and (23) allow the number of free fitting parameters to be reduced.

As we shall show later in this work, the decay of the intermediate scattering function $F(k, \tau)$ at a particular wavevector can be accurately described by the superposition of two real exponentials (for the two diffusive decay modes) and one complex conjugate pair exponential (for the short time non-diffusive behavior). Therefore, in this work we will approximate the solution by retaining the first three terms in Eq. (22), this results in:

$$
\begin{aligned}
F(k, t) & \approx A_{1} \exp \left(z_{1} t\right)+A_{2} \exp \left(z_{2} t\right) \\
& +\left(A_{3}^{\prime} \pm i A_{3}^{\prime \prime}\right) \exp \left(\left(z_{3}^{\prime} \pm i z_{3}^{\prime \prime}\right) t\right) .
\end{aligned}
$$

In line with usual experimental analysis, we can refer to the first two decay modes as the short-time and long-time diffusion modes with effective short-time $D_{s}$ and longtime $D_{L}$ diffusion coefficients. The complex exponential can also be simplified into a damped cosine form. Doing this results in

$$
\begin{aligned}
F(k, t) & \approx A_{s} \exp \left(-k^{2} D_{s} t\right)+A_{L} \exp \left(-k^{2} D_{L} t\right) \\
& +A_{d} \exp (\alpha t) \cos (-\beta t+\phi) .
\end{aligned}
$$

where all coefficients are wavevector dependent and we have included a factor of $k^{2}$ in the real exponentials. Eq. $(25)$ is the final form that is fitted to the simulation data.

Fitting Eq. (25) to simulation data requires optimization of 8 free fitting parameters. But, this number can be reduced by constraining the solution using the relationship given in Eq. (23) for $m=1$ and 3. Also, the normalization condition of $F(k, t=0)=1$ allows an additional variable to be eliminated, resulting in the total elimination of 3 free parameters, thus reducing the computational complexity of the problem.

The fitting of the multiexponential model was performed by means of a program run in the Matlab [41] environment, carried out by a built-in nonlinear least squares algorithm. The implementation of the 3 constraints was done by using the inbuilt solve function to give expressions for the chosen dependent parameters in terms of the independent ones.

\section{SIMULATION METHODS}

The pair potential and parameters for our simulations are identical to previous work [25] but we will include a brief explanation here. We modeled the colloidal particle and solvent using a Weeks-Chandler-Andersen (WCA) potential (a shifted and truncated Lennard-Jones potential) which is modified to include a hard-core. The potential takes the form

$\phi\left(r_{i j}\right)= \begin{cases}\infty & \text { if } r_{i j} \leq c_{a b} \\ \epsilon\left[\left(\frac{\sigma}{r_{i j}-c_{a b}}\right)^{12}-\left(\frac{\sigma}{r_{i j}-c_{a b}}\right)^{6}\right]+\epsilon & \text { if } c_{a b}<r_{i j}<c_{a b}+2^{1 / 6} \\ 0 & \text { otherwise }\end{cases}$ where $r_{i j}$ is the centre-to-centre distance between the particles $i$ and $j, \epsilon$ is the depth of the potential well, $\sigma$ is the nominal length scale of the potential (in this work $\epsilon=\sigma=1$ and therefore all results are given in reduced units). The $c_{a b}$ parameter introduces a hard-core to the potential where $a$ and $b$ denote the two interacting species. This creates an excluded region which is used to increase the size of the colloidal particle relative to the solvent. A diagram of the potential is shown in Fig. 1.

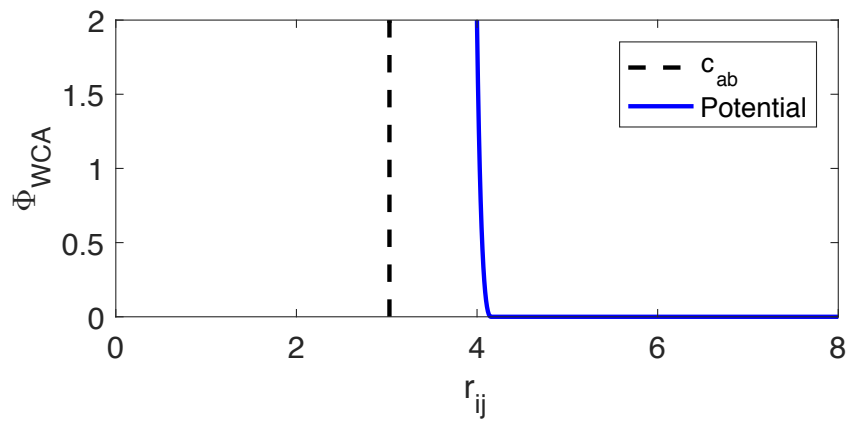

FIG. 1. Diagram of pair potential between colloidal particles with $\epsilon=\sigma=1.00$ and $c_{a b}=3.03$. 
In this work we keep the values of the $c_{a b}$ fixed for the colloid-colloid $\left(c_{11}\right)$ and solvent-solvent $\left(c_{22}\right)$ interactions at 3.03 and 0.000 respectively. The $c_{12}$ parameter for the colloid-solvent interaction is also set to 0.000 to remove the depletion effects that are inherent in systems with different sized particles. In usual hard-sphere simulations the diameter of the particle is clear, but in this work we are using a hard-core plus a WCA repulsive potential. This WCA repulsive potential adds an extra diameter that is not clearly defined.

Previous work has been done by Hess et al. to determine expressions for the effective diameter of WCA particles as a function of temperature [42]. These authors found that the method which gave the best agreement with the MD results was to define the effective diameter $d$ to be when the interaction potential is equal to Boltzmann's constant times the temperature $\phi(d)=k_{B} T$. At the reduced temperature of 1.0 used in this work, this gives an extra diameter of $\sigma$ to the particles due to the WCA repulsion. This gives the colloidal particles an effective diameter of 4.03 times the diameter of the solvent particles.

The mass of the colloidal particle was set with the goal of making it approximately neutrally buoyant in the solvent. The mass needed to do this was calculated in the same way as McPhie [43], which for a size ratio $d_{1} / d_{2}$ of 4.03 gave a mass ratio $m_{1} / m_{2}$ of 50 . Therefore, we used a mass of 1.0 for the solvent particles and a mass of 50.0 for the larger particles. This size and mass ratio has been shown to be large enough for the larger particle to behave as a Brownian particle in the solvent [44].

All simulations were run using the MD package LAMMPS [45] and the results were post-processed using in-house code. Simulations at each packing fraction were done under NPT conditions at a reduced temperature of 1.00 and reduced pressure of 7.85. The time integration scheme used follows the time-reversible measurepreserving Verlet integrator derived by Tuckerman et al. [46] with a time step of 0.005 . The temperature is held fixed using a Gaussian thermostat while the pressure is held fixed using a Nose-Hoover type barostat, both used a damping parameter of 10 . This was done in order to better replicate the experimental conditions of a real colloidal suspension. All simulations were done with a total of 108,000 particles, except for one larger system which was done in order to calculate data for small $k$ values of the intermediate scattering function at the packing fraction of 0.49 . This simulation had 864,000 particles. Table I gives the exact number of particles used in the simulations, along with the average volume and calculated packing fraction. The packing fraction $\Phi$ was calculated from

$$
\Phi=\frac{\pi N_{c} d^{3}}{6 V}
$$

where $d$ is the diameter of the colloidal particles $(d=$ 4.03 for all colloidal particles). In our previous work we showed that calculating $\Phi$ for this system based on Eq.
(26) resulted in a phase behavior that matched closely to that of a single component HS system [25].

TABLE I. Number of solvent particles $N_{s}$, colloidal particles $N_{c}$, average volume $\langle V\rangle$ and packing fraction $\Phi$ for the systems studied.

\begin{tabular}{lllll}
\hline \hline $\begin{array}{l}\text { System } \\
\text { No. }\end{array}$ & $N_{s}$ & $N_{c}$ & $\langle V\rangle$ & $\Phi$ \\
\hline 1 & 107,967 & 33 & 128,606 & 0.01 \\
2 & 107,652 & 348 & 128,550 & 0.09 \\
3 & 107,325 & 675 & 128,597 & 0.18 \\
4 & 106,988 & 1,012 & 128,600 & 0.27 \\
5 & 106,650 & 1,350 & 128,887 & 0.36 \\
6 & 106,313 & 1,687 & 129,085 & 0.44 \\
7 & 106,145 & 1,855 & 129,315 & 0.49 \\
8 & 849,160 & 14,840 & $1,028,848$ & 0.49 \\
\hline
\end{tabular}

In the following sections we will be mostly calculating properties of the larger particles (meant to represent the colloidal particles). Because of this, subscripts designating species will be dropped and unless otherwise stated the properties being measured are for the colloidal particles only (i.e. the colloidal particle intermediate scattering function $F_{11}(k, \tau)$ will just be represented as $F(k, \tau)$ etc).

\section{RESULTS}

\section{A. Velocity autocorrelation function and self diffusion coefficient}

Fig. 2 displays results for the absolute value of the colloidal particle velocity autocorrelation function $|C(\tau)|$. It is displayed on a log-log scale as the decay of $C(\tau)$ covers multiple orders of magnitude across a number of decades in time. The curves shown cover a large range of packing fractions from a dilute system $(\Phi=0.09)$ to a system at a packing fractions slightly lower than the freezing point $(\Phi=0.49)$. C $(\tau)$ has been calculated previously for this model [25], but we include it here again as the quality of the data has been greatly improved which allows a more thorough comparison with pure HS simulation results, as well as experimental data.

The most thorough study to date on the velocity autocorrelation function for a pure HS system (no solvent) was done by Williams and coworkers [47, 48]. They were able to observe the $-3 / 2$ power law long-time tail (which is the manifestation of diffusing transverse modes) and velocity reversals (which are the result of damped compression modes). For stable fluids, velocity reversals were only observed for high packing fractions $(\Phi \geq 0.44)$. In Fig. 2 we also observe velocity reversals (indicated by the spike downward on the $\log (|C(\tau)|)$ plot where $C(\tau)$ becomes negative). The reversals are seen for all $\Phi$ shown 
$(0.09 \leq \Phi \leq 0.49)$, with the time it takes for the reversals to occur decreasing as $\Phi$ increases.

Both the pure HS (without solvent) and our model (with solvent) can be compared with available experimental data. It is very difficult to determine experimentally the complete behaviour of $\mathrm{C}(\tau)$. The initial decay of $\mathrm{C}(\tau)$ (which gives the initial crossing through zero) is difficult to obtain accurately. Even so, reversals in $C(\tau)$ are observed at packing fractions as low as $\Phi=0.289$ in experimental colloidal suspensions [50] where the long-time decay of $C(\tau)$ occurs from below zero. This is consistent with the results from our model, but disagrees with the results from the pure HS system. The mismatch between the sign of $C(\tau)$ at low to moderate $\Phi$ for the single component HS fluid was pointed out in our previous work [25], indicating that the current model possesses dynamics which better resemble that of an experimental colloidal suspension. A possible reason why the pure HS model fails to predict the reversals at low to moderate packing fractions is because it neglects the solvent, and therefore ignores hydrodynamic effects and momentum transfer via the solvent, both of which could cause velocity reversals.

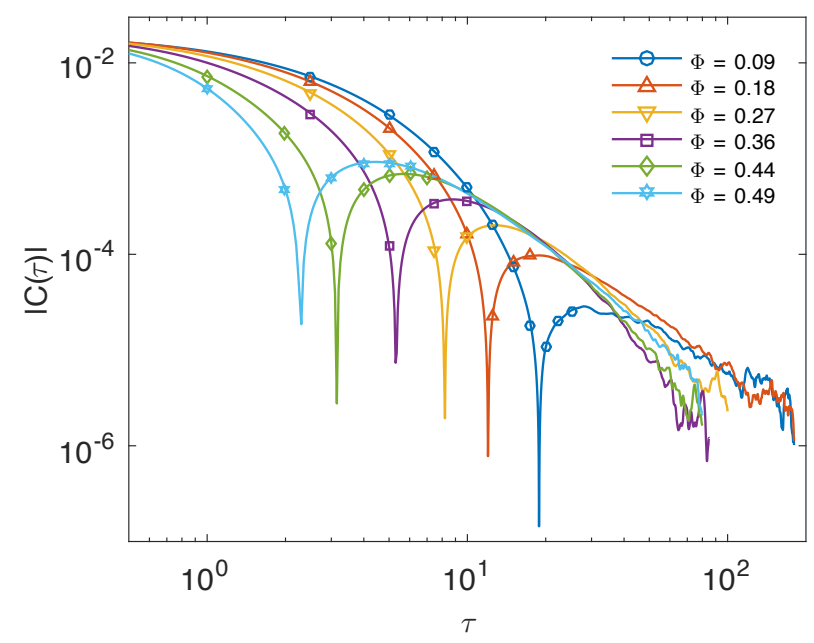

FIG. 2. Plot of the colloidal particle velocity autocorrelation function $\mathrm{C}(\tau)$ at packing fractions leading up to the freezing point. The points where $C(\tau)$ cross zero may be seen as a sharp spike downward in this log-log graph.

However, current data for this model suffers from the same limitations as experiment, in that the power-law long-time decay of $\mathrm{C}(\tau)$ falls within the noise and cannot be observed. This is due to the smaller number of colloidal particles and the much larger mass, which results is smaller velocities.

The colloidal particle self diffusion coefficient $D_{s}$ is related to the ability of an individual colloidal particle to diffuse through the liquid. This property was calculated from Eq. (4) and is shown in Fig. 3. We have normalized the data by dividing by the dilute limit value of $(5.93 \pm 0.05) \times 10^{-2}$. The maximum value of $D_{s}$ occurs

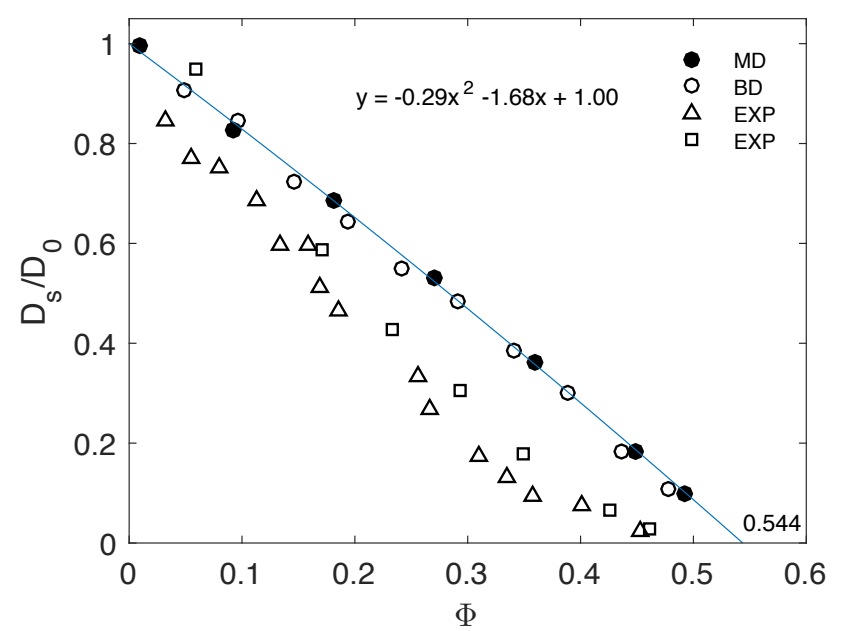

FIG. 3. Plot of the normalized colloidal particle self diffusion coefficient $D_{s} / D_{0}$ against packing fraction. Filled circles: our MD data; open circles: BD data of Moriguchi [51]; triangles: experimental data of van Megen and Underwood [52]; squares: experimental data of van Blaaderen et al. [53]. Quadratic line of best fit to MD data with the numerical value of $\mathrm{x}$-intercept (at 0.544) also shown.

in the dilute limit as the motion is not hindered by direct interactions with other colloidal particles.

As the packing fraction increases, the ability of a single colloidal particle to diffuse through the fluid is hindered. This results in a decrease of the self diffusion coefficient. In Fig. 3 it can be seen that results from previous Brownian Dynamics simulations by Moriguchi [51] over-predict $D_{s}$ when compared to experimental systems $[52,53]$. They were able to show that an inclusion of a hydrodynamic correction brought $\mathrm{BD}$ and experiment into agreement.

The current model model also over-estimate $D_{s}$ (as seen in Fig. 3) even though the solvent (and therefore hydrodynamic effects) are included. This disagreement could be due to the modified interaction parameters used to reduce the depletion effects $\left(c_{12}=0\right)$. By reducing the $c_{12}$ parameter, the volume of the colloid as seen by the solvent is much lower than the volume used in the calculation of the packing fraction. This may have the effect of diluting the hydrodynamic interactions.

To see where $D_{s}$ extrapolates to zero (where the free movement of the colloidal particles is completely removed), a second order quadratic function was fitted to the data and the $x$-intercept was calculated. This is found to occur at $\phi=0.544 \pm 0.010$ which is within the uncertainty of the melting point $\phi=0.545$ where the hard-spheres fully crystallise. This indicates that the diffusive motion of the colloids is frozen out at this point.

The self diffusion coefficient of real colloidal suspensions can be measured in DLS via the self intermediate scattering function [55]. From the self intermediate scattering function the mean squared displacement can be 
determined, which is related to the so called short and long time self diffusion coefficients. Interestingly, the normalised self diffusion coefficient of an experimental glass forming system has been shown to go to zero at its glass transition [55], rather than the melting point.

\section{B. Mutual Diffusion Coefficient}

In this model (as in real colloidal suspensions) the solvent is explicitly present and so we have a binary fluid with a single mutual diffusion coefficient [32]. Calculation of $D_{m}$ (definition given in Eq. (6)) requires accurate values of the phenomenological coefficient $L_{11}$ and the thermodynamic factor $\partial \mu_{1} / \partial c_{1}$. We will outline here how these two quantities were calculated.

The phenomenological coefficient $L_{11}$ was calculated from the mass flux correlation function of the colloidal particles defined in Eqs. (7), (8) and (9). The mass flux correlation function was calculated every 5 time steps out to a maximum delay time of 25,000 timesteps. Numerical integration with the trapezoid rule was performed on the correlation function and the integrals were found to converge. The values of the integral for each packing fraction are shown in Fig. 4. $L_{11}$ is shown to increase as $\Phi$ increases, but the rate of increase (given by the slope of the plot) decreases as the freezing point is approached. At the freezing point the slope is observed to almost go to zero, and $L_{11}$ appears to plateau.

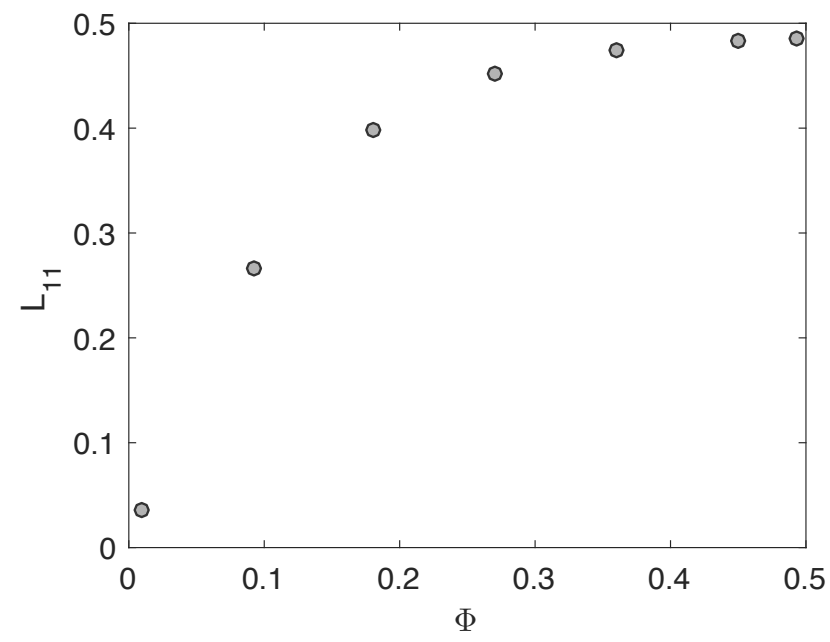

FIG. 4. Plot of the phenomenological coefficient $L_{11}$ calculated from the integral of the colloidal particle mass flux autocorrelation function.

The second quantity that needs to be calculated is the thermodynamic factor $\partial \mu_{1} / \partial c_{1}$ which is related to the integrals of the radial distribution functions $G_{\alpha \beta}$. There are a number of different methods available to calculate the thermodynamic factors from MD simulation, such as through the numerical integration of the radial distribution functions [35], the Widom test particle insertion method [56], from density fluctuations of a smaller subsystem embedded in simulation box [57] or through the static structure factors [37]. In this work we obtained the thermodynamic factor through the static structure factors by using Eq. (21). It is important to note that Eq. (21) differs from the expression given by Nichols and Wheeler [58] who previously proposed a simular method. This is because the definition of the thermodynamic factors are different, but both methods produce the same value for $D_{m}$ when combined with their complete expression.

As an example of how the $S_{\alpha \beta}(k \rightarrow 0)$ values was calculated for each packing fraction, Fig. 5 shows a plot of the static structure factors $S_{11}$ (colloid-colloid), $S_{12}$ (colloidsolvent) and $S_{22}$ (solvent-solvent) of the system at a packing fraction of 0.49 . The data was plotted against $k^{2}$ as the $S(k)$ should be an even function of $k$, and 5 th order polynomials in $k^{2}$ were fitted to the low- $k$ data. Although it is possible that $S(k)$ could be a non-analytic function of $k$, and could therefore also depend on odd or fractional powers of $|k|$, we saw no evidence of this in our data. By extrapolating the polynomial in $k^{2}$ back to zero $k$ we were able to accurately determine $S_{\alpha \beta}(k \rightarrow 0)$ values.

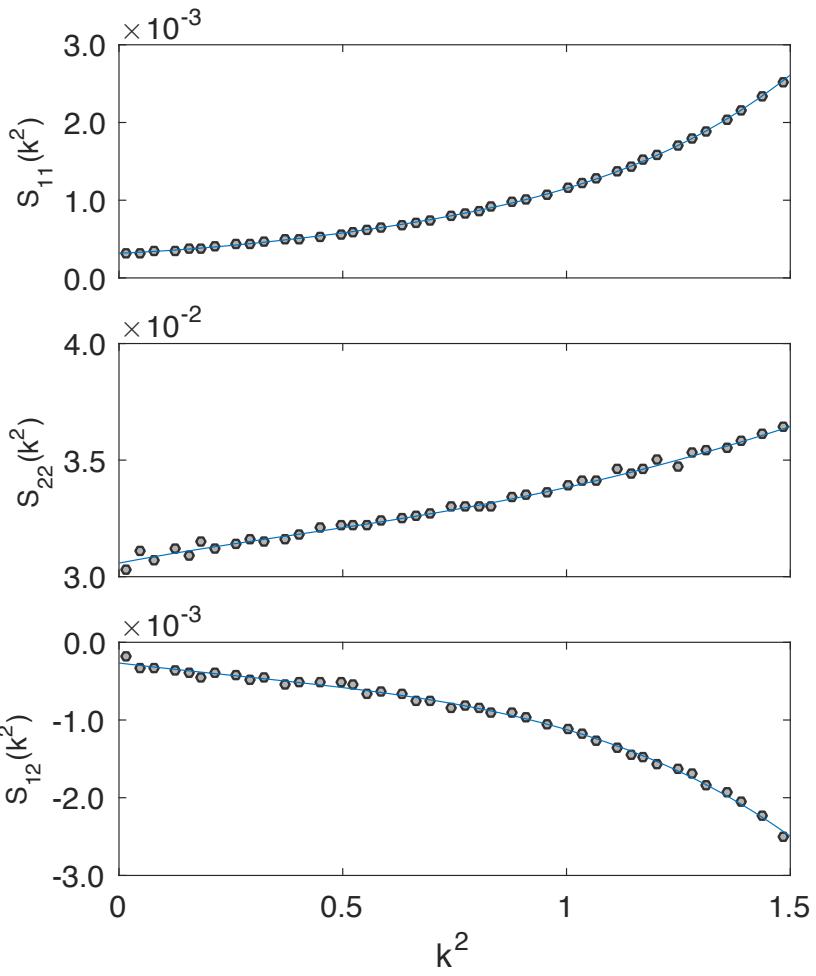

FIG. 5. Plot of the low-k behavior of the static structure factors: $S_{11}$ (colloid-colloid), $S_{12}$ (colloid-solvent) and $S_{22}$ (solvent-solvent) of a system at a packing fraction of 0.49 . A 5 th order polynomial line of best fit was used to obtain $S_{\alpha \beta}(k \rightarrow 0)$ values.

This was done for a range of packing fractions from an extremely dilute fluid $(\Phi=0.01)$ up to a high density 
fluid ( $\Phi=0.49$ ). The highest density fluid is slightly below the freezing point that occurs at $\Phi_{F}=0.494$. The values of $S_{\alpha \beta}$ calculated for each packing fraction were used in (21) to calculate $G_{\alpha \beta}$, which are shown in Fig. 6. The quantities $G_{12}$ and $G_{22}$ have almost negligible contribution to the calculation of the thermodynamic factor $\partial \mu / \partial c_{1}$ as they are two orders of magnitude smaller than $G_{11}$. The magnitude of $G_{11}$ decreases as $\Phi$ increases, and this results in an increase in $\partial \mu / \partial c_{1}$ as the two are inversely related (from Eqs. (15) and (16)). The values of
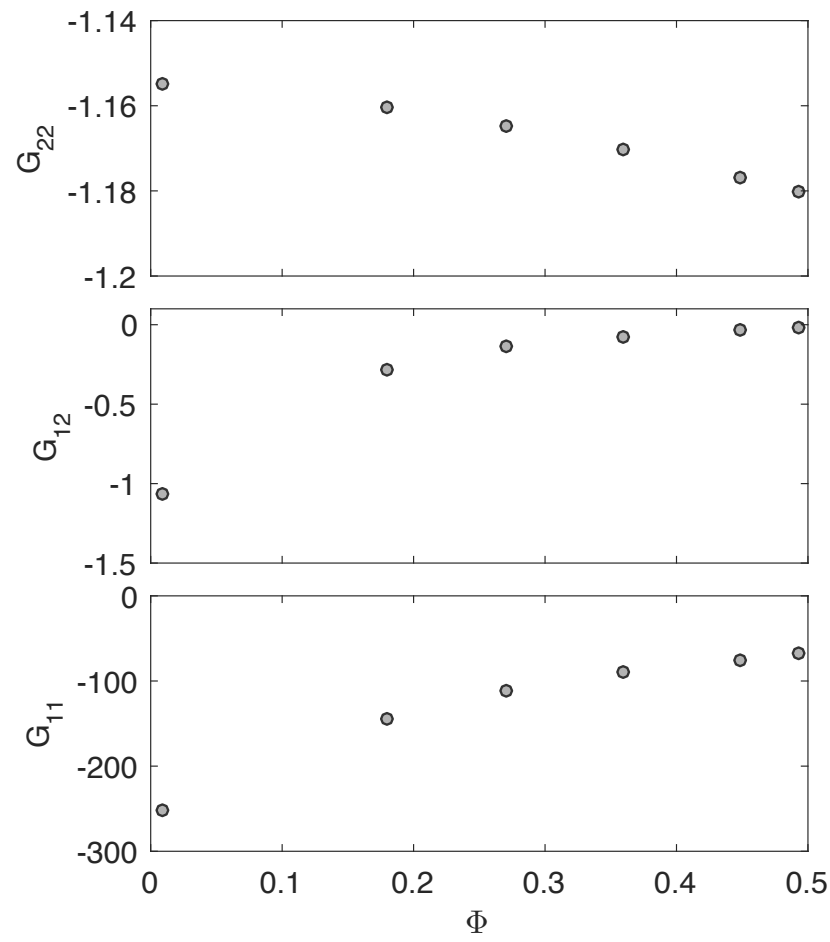

FIG. 6. Plot of the radial distribution function integrals of $G_{11}$ (colloid-colloid), $G_{12}$ (colloid-solvent) and $G_{22}$ (solventsolvent) at packing fractions $\Phi$ leading up to the freezing point.

$G_{\alpha, \beta}$ were used in Eqs. (15) and (16) to calculate $\partial \mu / \partial c_{1}$ for each $\Phi$, these are plotted in Fig. 7. The thermodynamic factor shows a decrease at low concentration, but increases greatly on the approach to the freezing point.

Using the values given for $L_{11}$ and $\partial \mu_{1} / \partial c_{1}$, the mutual diffusion coefficient $D_{m}$ was calculated and is shown in Fig. 8. The mutual diffusion coefficient $D_{m}$ shows an increase on the approach to the freezing point. From the contributions of $L_{11}$ and $\partial \mu / \partial c_{1}$ in Fig. 4 and 7 we see that this increase in $D_{m}$ results mainly from the $\partial \mu / \partial c_{1}$ contribution rather than from $L_{11}$, as the latter quantity approaches a plateau near the freezing point.

Eq. (14) predicts that the low- $k$ values of the intermediate scattering function will decay faster at higher packing fractions, even as the ability of the individual colloidal particles to diffuse through the liquid decreases on the

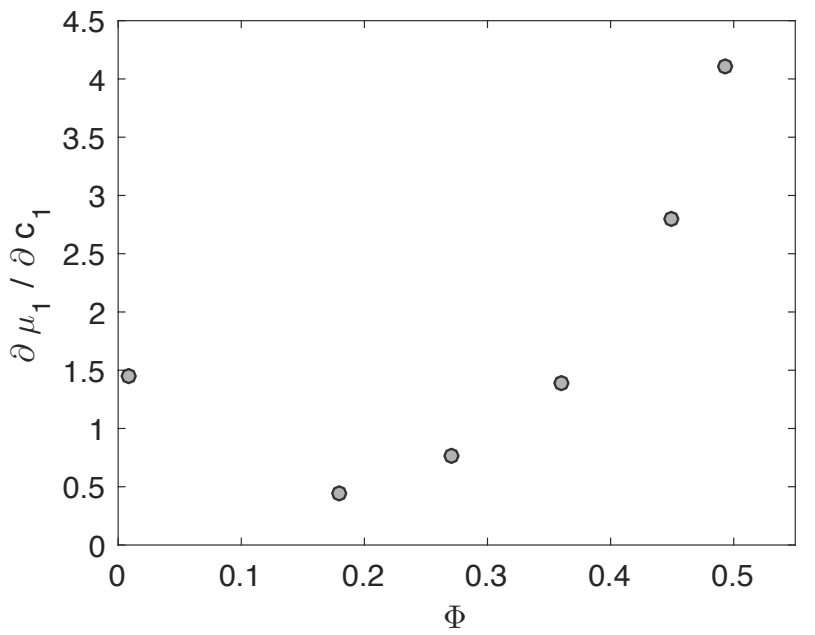

FIG. 7. Plot of the thermodynamic factor $\partial \mu / \partial c_{1}$ at packing fractions $\Phi$ leading up to the freezing point.

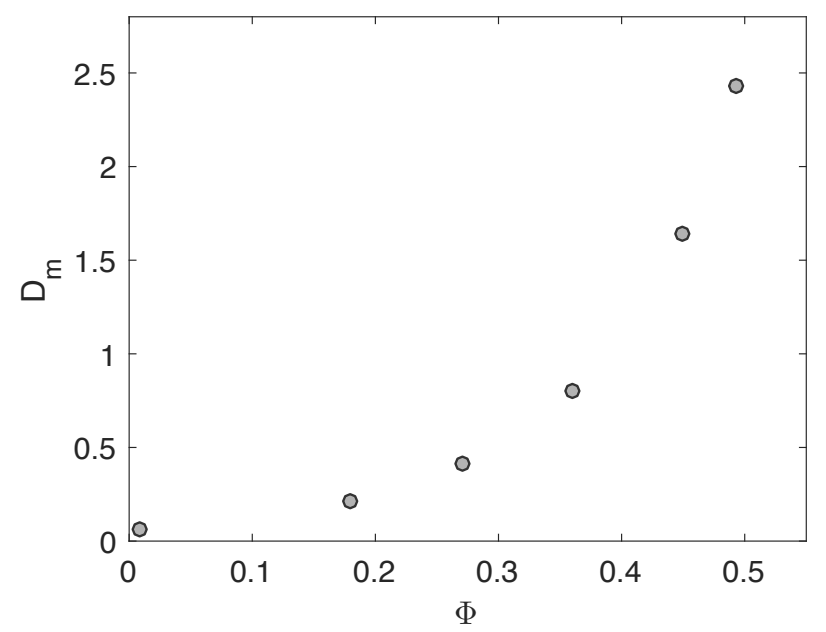

FIG. 8. Plot of the mutual diffusion coefficient $D_{m}$ at packing fractions $\Phi$ leading up to the freezing point.

approach to the freezing point. This results in a broadening of the decay times between the small wavevector and large wavevector decay, which will be shown later.

\section{Intermediate Scattering Function}

In this section, calculations of the colloidal particle intermediate scattering function $F(k, \tau)$ will be shown for the higher packing fractions $\Phi$ leading up to the freezing point. The systems studied are described in Table I. The wavevectors studied were those consistent with the periodic boundaries of the simulation box (given in Eq. (3)) up to $n_{1}=n_{2}=n_{3}=15$. In this section we will report wavevectors in the dimensionless form $k d$ where $d$ is the diameter of the colloidal particles. This allows di- 
rect comparison with corresponding $k d$ values measured in light scattering experiments.

To show the change in behavior of $F(k, \tau)$ leading up to the freezing point, we have displayed the calculations of $F(k, \tau)$ at the packing fractions of $0.27,0.36$ and 0.49 in Fig. 9 (data given as symbols). The wavevectors shown have been chosen to cover a range from the lowest wavevector allowed (consistent with periodic boundary conditions) to just above the colloidal particle static structure factor peak. Plots of $\ln (F(k, \tau))$ against $k^{2} \tau$ which exhibit regions of constant slope imply exponential decays in time, or simple Brownian diffusion. The fits of the form given in Eq. (25) are also shown in Fig. 9 (lines).
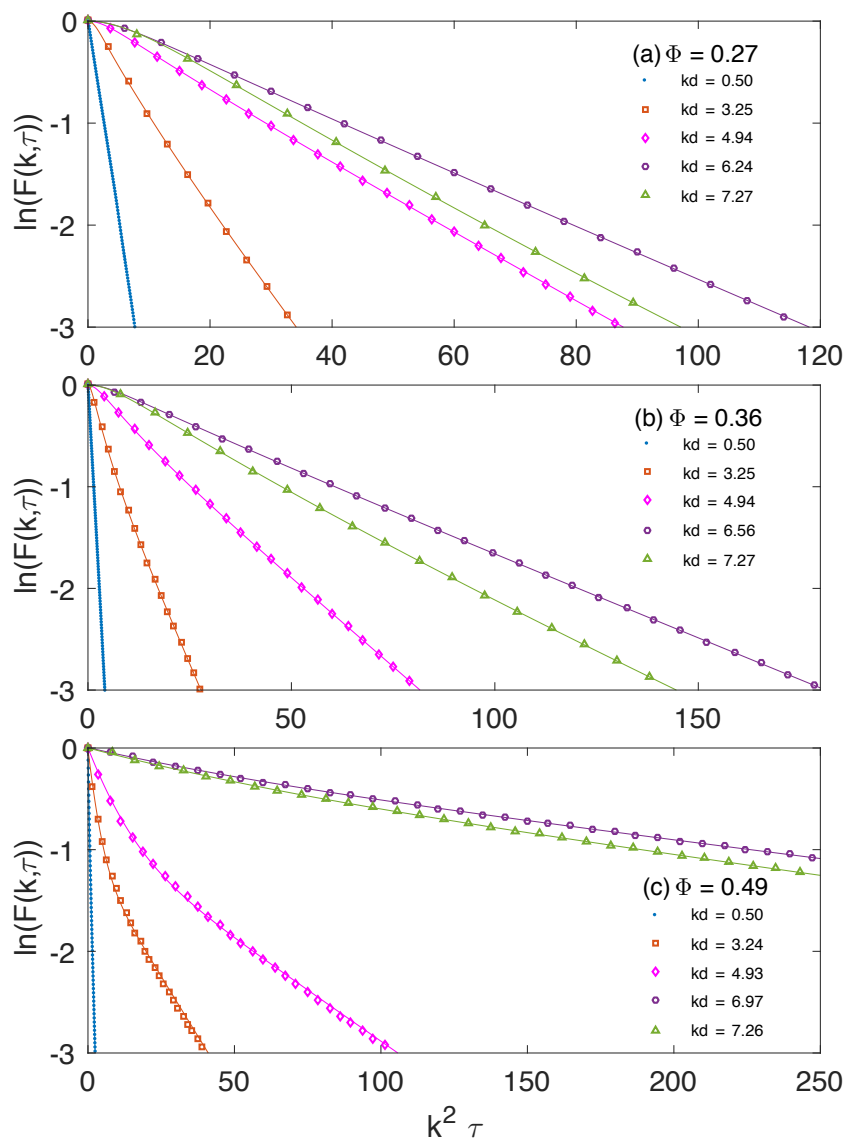

FIG. 9. Plot of the $\ln (F(k, \tau))$ against $k^{2} \tau$ for packing fractions of (a) 0.27 , (b) 0.36 and (c) 0.49 for the $k d$ values given in the legend. The data for the lowest $k d$ (dots) corresponds to the lowest $k d$ possible to calculate based on the box dimensions, and the second largest value (circles) corresponds to the colloidal particle static structure factor peak.

For an extremely dilute colloidal suspension, the effects of direct colloidal particle interactions are negligible due to the large average distances between neighboring particles. This means their dynamics are governed by random collisions with the surrounding solvent, so they effectively move as Brownian particles. The $F(k, t)$ in these dilute suspensions $(\Phi \approx 0.01)$ decay as a single exponential with a $k$-independent diffusion coefficient. This is also seen in light scattering experiments [7] (though there is a small $k$ dependence in the diffusion coefficient which is associated with polydispersity) and in our previous work using the current computer simulation model [25].

Calculations for $F(k, t)$ of a system at $\Phi=0.27$ are shown in Fig. 9a. The decay for all wavevectors is (almost) linear, just as in a dilute system, but the slope of the decay changes with each new $k$. This implies the existence of time-independent diffusion with a wavevector dependent diffusion coefficient $D(k)$. With the plot displayed in this way, the lowest $k$-value has the largest slope, indicating it has the largest effective diffusion coefficient.

As the wavevector is increased, the slope decreases up to the structure factor peak of $(k d)_{\max }=6.24$ (circles). At wavevectors above the structure factor peak we see a reversal in the trend, where the slope is seen to have increased at $k d=7.27$ (triangles) showing a minimum in the diffusion coefficient at the structure factor peak. It is well verified that in concentrated colloidal suspensions the effective diffusion coefficient has a minimum at the peak in $S(k)[7,9]$. This minimum reflects the fact that the strong fluctuations that occur at the peak will decay slower than the weak fluctuations away from the peak. It should be noted though that in order to properly fit the data for $\Phi=0.27$, the second diffusive mode was still needed (though it was very weak).

Fig. 9 also reveals the change in behavior of $F(k, t)$ as the packing fraction increases towards the freezing point. At $\Phi=0.36$ (Fig. 9b) the slope is also seen to decrease as the wavevector increases, and has a minimum at the structure factor peak. Interestingly, the spread in the slopes has been greatly increased compared to $\Phi=0.27$. The slope at the lowest wavevector $(k d=0.50)$ has increased compared to $\Phi=0.27$ at the same wavevector (note the change in scale on the $\mathrm{x}$-axis), while the slope at the structure factor peak $(k d=6.56)$ has decreased. The increasing slope at low wavevectors is the result of the increase in the mutual diffusion coefficient (shown in Fig. $8)$ as it is this coefficient that governs the decay of $F(k, t)$ in the low wavevector limit. The decay of $\ln (F(k, t))$ for all wavevectors at $\Phi=0.36$ is also not quite linear, with a slight non-linearity of the line of best fit most clearly seen at $k d=4.94$ (diamonds). This is indicative that the effective diffusion coefficient is becoming time dependent. Just below freezing at $\Phi=0.49$ (Fig. 9c) the two decay modes are clearly seen, with a significant non-linearity occurring at wavevectors between zero and the structure factor peak.

This method of fitting Eq. (25) to find the behavior of the short-time and long-time diffusion coefficients is similar to that done to experimental results by Orsi et. al. [27]. The multiexponential analysis procedure that we performed on our data allows the contributions from the individual terms in Eq. (25) to be separated and studied. To better quantify the behavior of the modes and their wavevector and packing fraction dependence, 

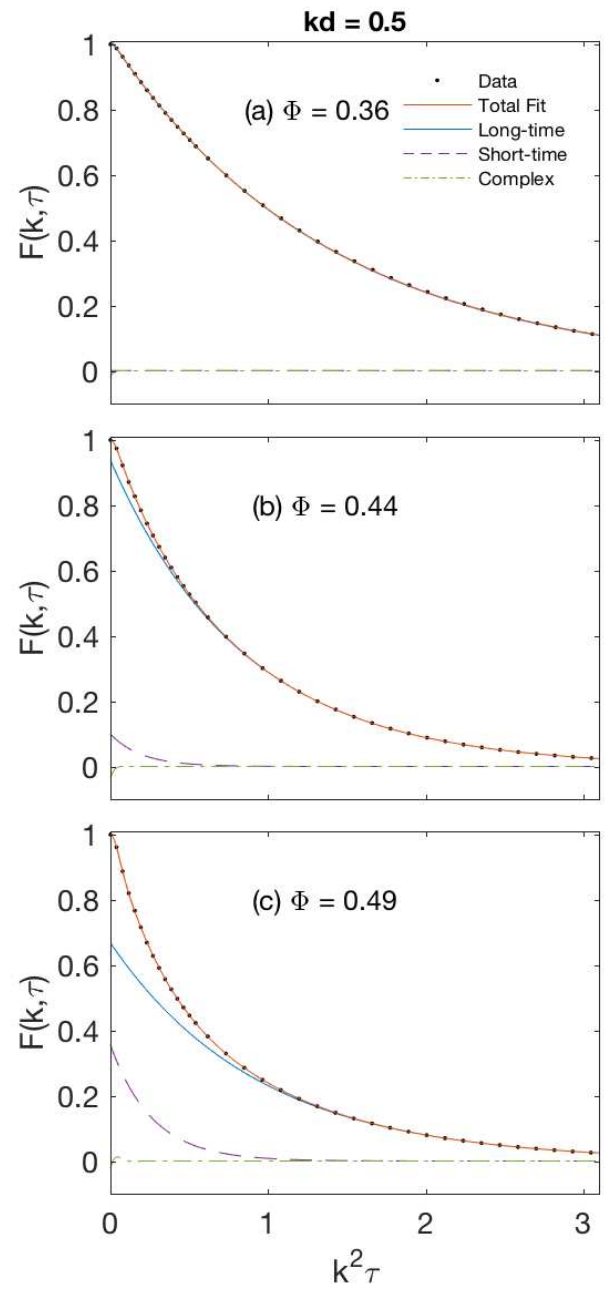

$\mathrm{kd}=3.5$

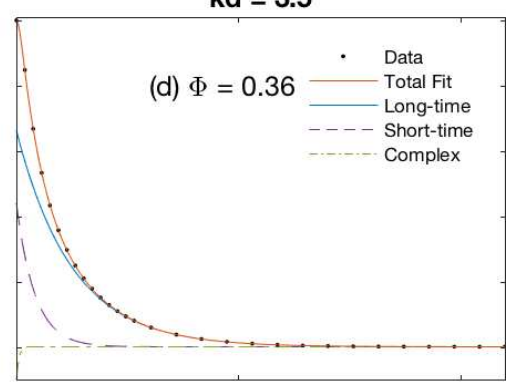

(e) $\Phi=0.44$
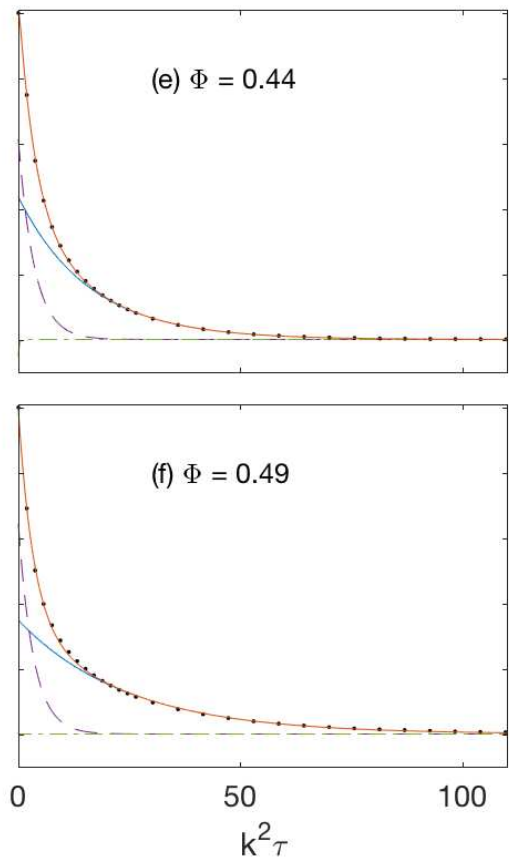
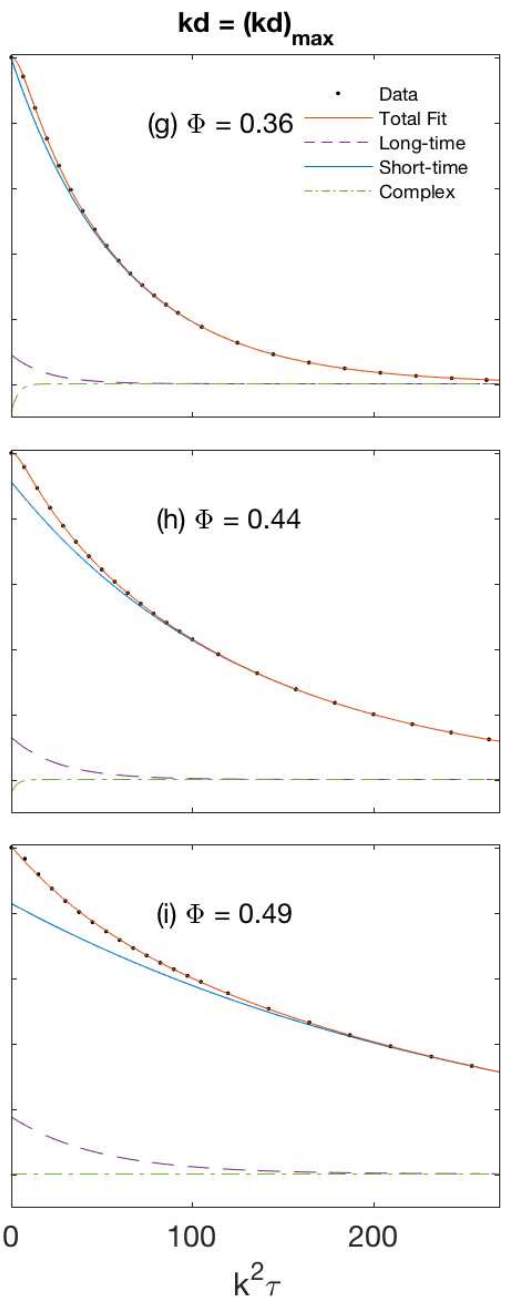

FIG. 10. Plot of $F(k, t)$ data (symbols) for the three indicated packing fractions $\Phi$ together with the multiexponential fit described in the text (red solid line through the data points). The left frames [(a)-(c)] are at a low wavevector $(k d=0.5)$, the middle frames $[(\mathrm{d})$-(f)] are at a wavevector half-way to the structure factor peak $(k d=3.5)$ and the right frames $[(\mathrm{g})$-(i)] are at the wavevector that corresponds to the structure factor peak $(k d \approx 7)$. The various components of the fit function are also displayed separately according to the legend. Short-time and Long-time denote the real exponential terms ordered by increasing decay time. Complex denotes the sum of the two complex conjugate exponentials, amounting to a damped oscillatory function. For graphical clarity, not all available data points have been displayed.

we have plotted the total fit and the individual contributions in Fig. 10. This was done for the 3 highest $\Phi$ values, $0.36,0.44$ and 0.49 as all lower $\Phi$ exhibit mostly single exponential decay.

The third term in Eq. (25) is the sum of two complex conjugate exponentials, which gives a damped cosine function. This mode is labelled complex in Fig. 10, and is seen to be strongly damped and characterized by a very low amplitude. This mode mainly determines the behavior of $F(k, \tau)$ at very short times where non-exponential decay is observed. We observe non-exponential behavior at short times as the dynamics transition from atomic to Brownian motion. The size and mass ratio compared to the solvent is 4.03:1 and 50:1 respectively, rather than being effectively infinite as it would be for a real Brownian particle. Therefore, at very short time delays the behavior is not diffusive, though this non-diffusive mode quickly decays to zero leaving the two diffusive modes to dominate.

The two diffusive modes (the two real exponential terms in Eq. (25)) show interesting and complex dependence on packing fraction and wavevector. The left frames [(a)-(c)] of Fig. 10 show the decay at a very low wavevector $(k d=0.5)$. In this low-wavevector limit, $F(k, \tau)$ is expected to approach a single exponential as the wavelengths being probed approach the macroscopic diffusive limit (infinite wavelength). This behavior is observed in the low- $k$ results for the packing fraction of $\Phi=0.36$ (Fig. 10a) where the dominant contribution comes from the long-time diffusive mode, and the short time mode has an amplitude that is near zero.

For the higher packing fractions in the low- $k$ region 
(frames (b) and (c)), the long-time mode still dominates, but the short-time mode is non-zero and has a greater contribution. The second mode has a larger contribution because the wavevector being studied is not low enough to be in the macroscopic diffusive limit at these packing fractions. As the freezing point is approached, the minimum wavevector needed to achieve the macroscopic diffusive limit decreases. This minimum wavevector may even be unattainable when the fluid crystallizes, as the system would no longer be homogeneous. This may be an important indicator of the onset of crystallization in a system.
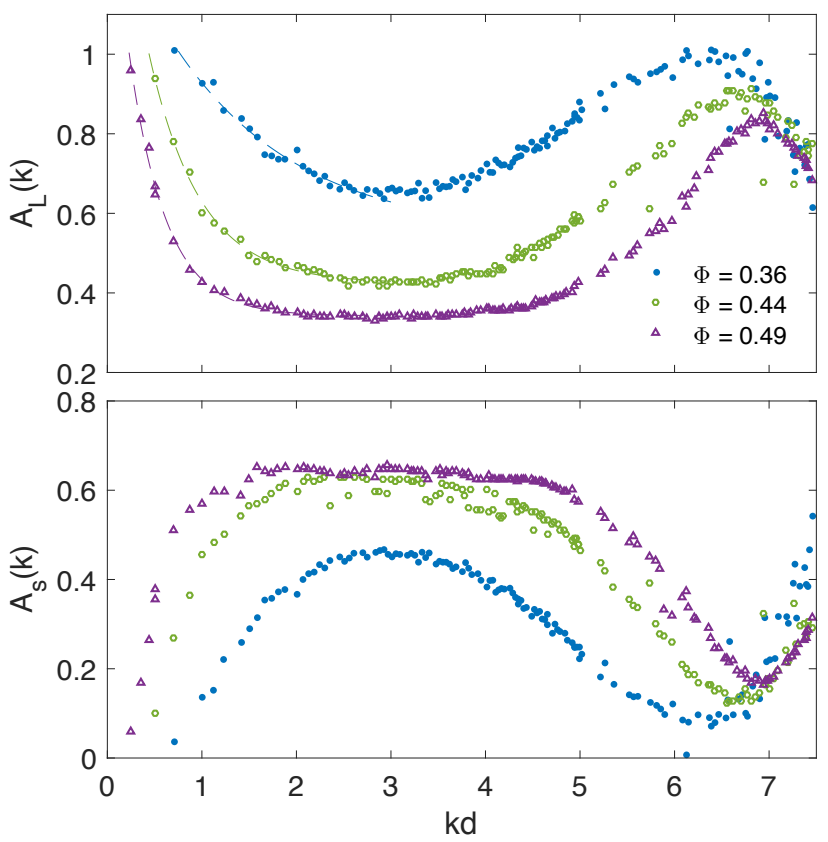

FIG. 11. Plot of the amplitudes of the long-time $D_{L}$ (top) and short-time $D_{S}$ (bottom) exponential decay modes for packing fractions of 0.36 (dots), 0.44 (circles) and 0.49 (triangles). Shifted exponential lines of best fit of the low- $k$ data for $D_{L}$ are also shown (dashed lines).

The middle frames [(d)-(f)] of Fig. 10 show the decay at $k d=3.5$ (which is roughly half-way to the peak in the structure factor). A comparison between data at these wavevectors and those at lower wavevectors shows there is a large decrease in the decay rate (note the expanded scale). This indicates that the effective short and long time diffusion coefficients have greatly decreased as the wavevector has increased. We also see an increase in the amplitude of the faster decay mode, showing that it has a larger contribution to the total fit.

Just as we observed at low wavevectors, the contribution of the short-time mode again increases as the packing fraction is increased. This can be seen by comparing the amplitude of the short-time mode in frames (d) and (f). At the larger packing fractions of $\phi=0.44$ and 0.49 the amplitude of the short-time mode is actually larger than the long-time mode (seen in frames (e) and (f)).
The right frames $[(\mathrm{g})-(\mathrm{i})]$ of Fig. 10 show the decay of $F(k, \tau)$ at $k d$ values that correspond to the main peak in the colloidal component structure factor. The effective diffusion coefficients continue to show a monotonic decrease as the packing fraction increases, shown by the increased decay time. As mentioned earlier, this is consistent with experimental results which show a minimum in the diffusion coefficients at the structure factor peak $[7,9]$. The amplitudes of the modes however do not show monotonic dependence on the wavevector. The long-time diffusive mode amplitude has increased in the approach to the structure factor peak, while the short-time one has decreased.

To display in more detail the complete behavior of the amplitudes and their dependence on packing fraction and wavevector, Fig. 11 shows the amplitudes of the two diffusive decay modes at the same packing fractions. As previously observed, at low wavevectors the amplitude of the long-time diffusive mode is almost unity, while the short-time one is almost zero. This indicates that the decay of $F(k, \tau)$ is close to a single exponential (as expected in the macroscopic diffusive limit).

Because the long-time mode still has a non-zero amplitude in the $k \rightarrow 0$ limit, it can be identified as a thermodynamic mode, where its decay rate in the macroscopic diffusive limit can be related to a thermodynamic quantity (later we show this to be the mutual diffusion coefficient). The short-time mode only exists for nonzero $k$, so can be identified as a kinetic mode which can not be related to any bulk property of the fluid. It can also be seen that the wavevector where the long-time diffusive mode amplitude goes to unity (or the short-time amplitude goes to zero) decreases as the freezing point is approached, and will most likely disappear when crystallization occurs.

To better quantify the packing fraction where the macroscopic diffusive limit can not be reached, a simple shifted exponential fit was done to the small wavevectors values of $A_{L}$ as shown in Fig. 11 (dashed lines). The $k d$ values where the exponential fits equal unity (when the macroscopic diffusive limit is reached) are displayed in Fig. 12 and are denoted by $(k d)_{M}$. These three $(k d)_{M}$ values are shown to have a linear dependence on $\Phi$, and an extrapolation of the linear fit to the $\Phi$-axis identifies the packing fraction of $\Phi \approx 0.546$ as when the diffusive limit disappears. This is extremely close to the melting point of $\Phi=0.545$ where HS systems completely crystallize and macroscopic diffusion is no longer possible.

The non-zero wavevector behavior of the amplitudes shows complex dependence. As seen in Fig. 11, the shorttime mode amplitude decreases to zero as $k d \rightarrow 0$, has a local maximum at $k d \approx 3$ and then a local minimum at the structure factor peak. It is interesting to note that although the local minimum at the structure factor peak depends on the packing fraction, the local maximum at $k d \approx 3$ does not. A complete explanation of the interesting non-monotonic behavior of the mode amplitudes is currently not known. 


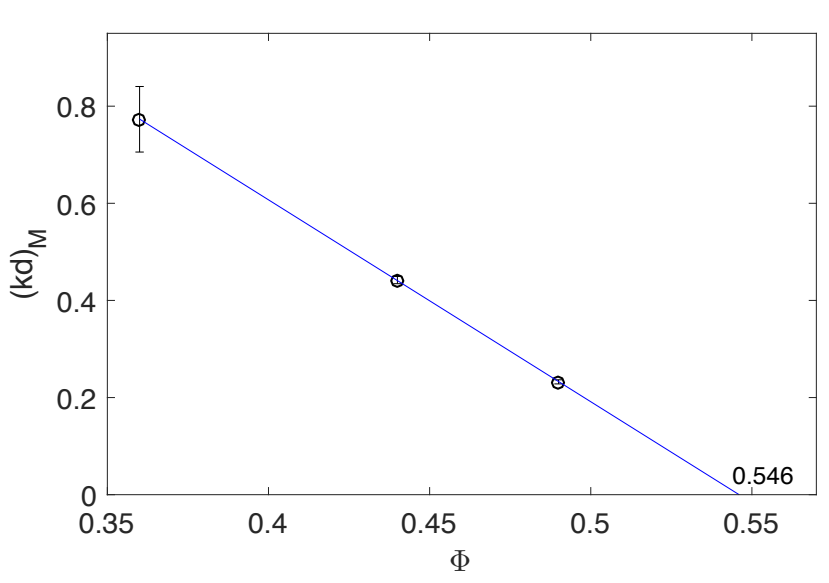

FIG. 12. Plot of the minimum wavevector $(k d)_{M}$ when single exponential decay of the intermediate scattering function is observed at each packing fraction $\Phi$. Error bars calculated based on the standard errors of fit coefficients in Fig. 11. Unweighted linear fit also shown with $x$-intercept of 0.546 .

Unlike the amplitudes, the effective long-time and short-time diffusion coefficients show a monotonic dependence on the wavevector between $k d=0$ and $(k d)_{\max }$. Previous DLS results [28] showed that the inverse of the effective diffusion coefficients correlate well with the static structure factor. To check that this is also true for this model colloid, the inverse of the short-time and longtime diffusion coefficients are plotted in Fig. 13, along with the scaled static structure factors.

In line with what is seen in experimental studies, the inverses of the diffusion coefficients roughly follow the static structure factor. Both have peaks at $(k d)_{\max }$, which corresponds to the value of the structure factor peak. Also, both approach zero in the $k \rightarrow 0$ limit. From this we can deduce that the diffusion coefficients (inverse of data in Fig. 13) have maxima at $k \rightarrow 0$ and minima at the structure factor peak (as expected).

The similarity of the shapes of $D_{L}$ and $D_{s}$ implies that they may be directly proportional, which would confirm the scaling law proposed by Segrè and Pusey [28]. In order to see if this scaling is observed with the current model, Fig. 14 shows the calculated ratio $D_{s} / D_{L}$ for all available $k$ vectors at the packing fractions of $0.36,0.44$ and 0.49 .

Segrè and Pusey [28] studied the intermediate scattering function of an experimental colloidal suspension over the wavevector range $2 \leq k d \leq 7.8$ and found the ratio $D_{s} / D_{L}$ was constant for $5 \leq k d \leq 7.8$ (around the structure factor peak). They found that by dividing $\ln (F(k, \tau))$ by $D_{s} k^{2}$, the decays at the wavevectors around the structure factor peak fell onto (roughly) a single master curve. Their work was done for a packing fraction of 0.465 .

Data for $\Phi=0.44$ (which is the closest $\Phi$ to that studied by Segrè and Pusey) is shown in Fig. 14. We see that the ratio is not constant, but has a wavevector depen-

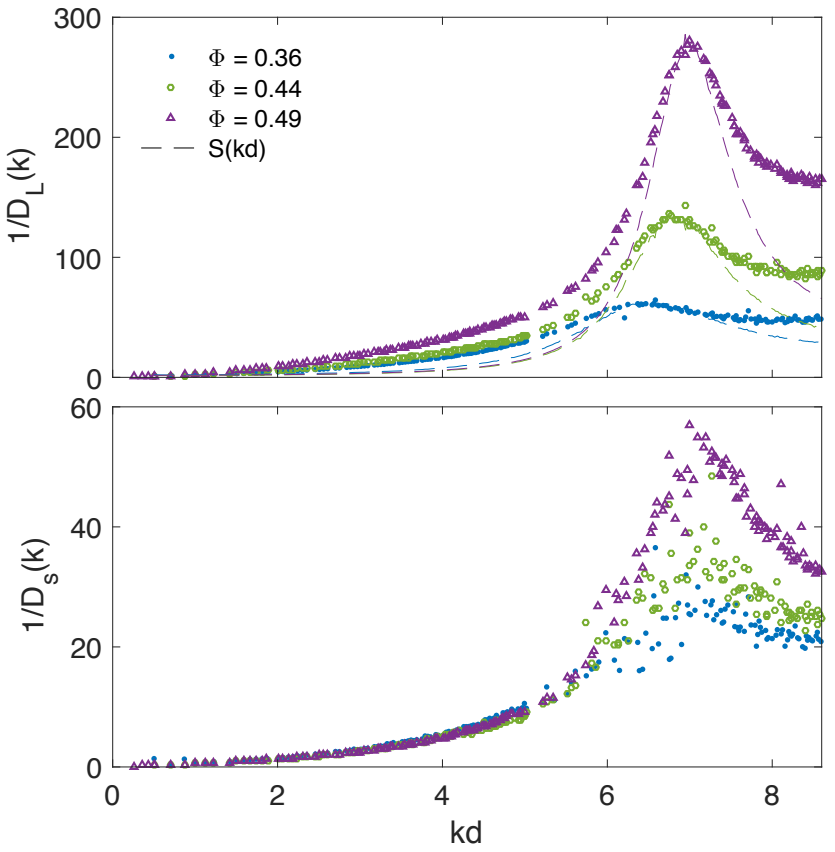

FIG. 13. Inverse diffusion coefficients of the long-time $D_{L}$ and short-time $D_{S}$ exponential decay modes for packing fractions of 0.36 (dots), 0.44 (circles) and 0.49 (triangles). Static structure factor data is also plotted (dashed lines) where the data has been scaled to match at the peaks.

dence. Even in the region studied by Segrè and Pusey $(5 \leq k d \leq 7.8)$, the ratio is not constant, showing a local maximum in this region.

The data for all packing fractions in Fig. 14 exhibit the same general shape, but the uncertainties are larger where the second diffusive mode is weak. Each packing fraction shows a slight peak around the position of the structure factor peak.

At $k d<5$ there is a slight increase in the ratio with a local maximum occurring at $\approx 2.6 \mathrm{kd}$. This is around the range where nonlinearity of $\ln (F(k, \tau))$ is most noticeable. This is partly due to the increase in the ratio, but also due to the increase in the amplitude of the faster mode in this region. At extremely small wavevectors $(k d<1.5)$ there is a rapid decrease in the ratio. This ratio could possibly extrapolate to 2 at zero wavevector, but this is impossible to determine from the current data. The average ratio over the whole range of wavevectors increases with packing fraction, showing the divergence of the two decay modes as the freezing point is approached.

Since the $k \rightarrow 0$ behavior of $F(k, t)$ should be given by the solution to the hydrodynamic equation (14), the decay should be a single exponential with a decay coefficient equal to the mutual diffusion coefficient $D_{m}$. To check this, we have plotted the low- $k$ values of $D_{L}$ in Fig. 15 (symbols) along with the mutual diffusion coefficients calculated from equilibrium MD using the GreenKubo and Kirkwood-Buff theory (arrows). The data for each $\Phi$ given in Fig. 15 has been shifted up by increments 

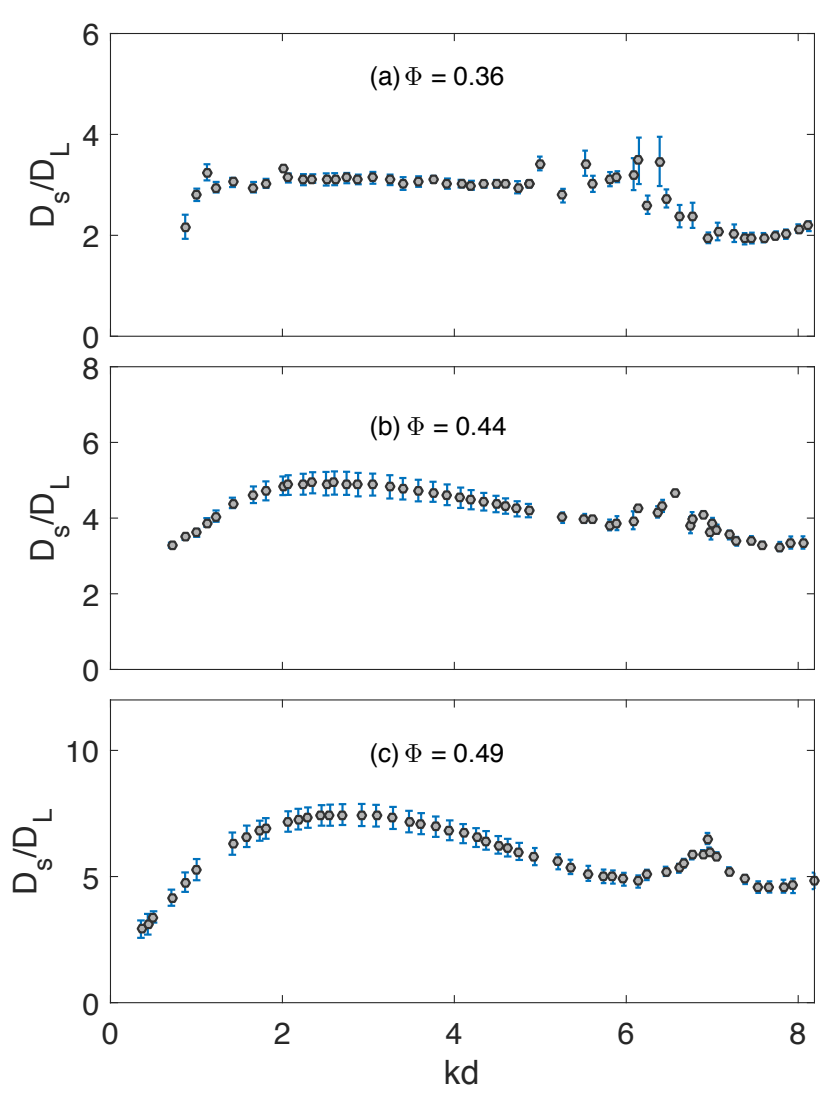

FIG. 14. Plot of the ratio $D_{s} / D_{L}$ for packing fractions of (a) 0.36 , (b) 0.44 and (c) 0.49 .

of 0.5 for clarity.

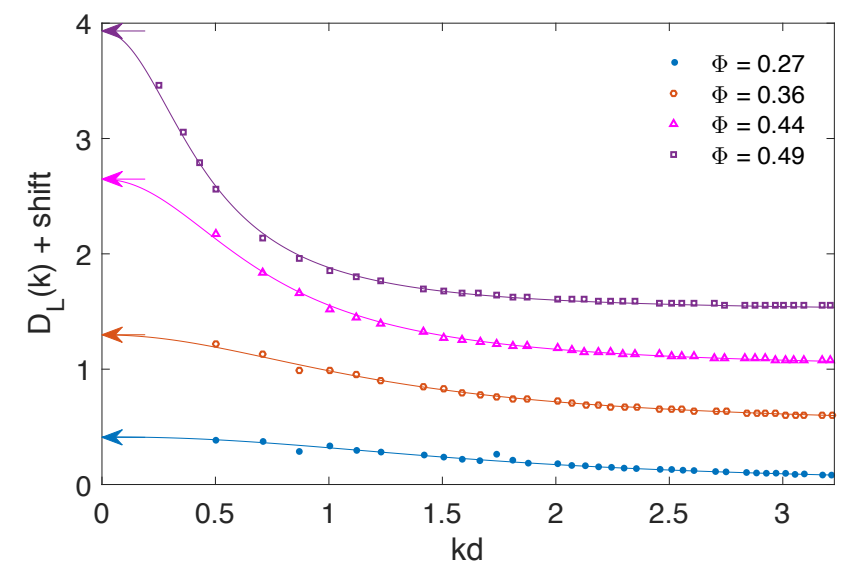

FIG. 15. Plot of low- $k$ values of the long time diffusion coefficient $D_{L}$ for packing fractions of 0.27 (dots), 0.36 (circles), 0.44 (triangles) and 0.49 (squares). Arrows on y-axis indicate the value of the mutual diffusion coefficients calculated for the corresponding packing fractions. Fits of the form given in Eq. (27) are also shown. Corresponding plots have been shifted up by increments of 0.5 for clarity.

Previous work done by Hansen et al. [59] showed that the wavevector dependence of the viscosity for a simple fluid could be fitted well with a Lorentzian type function with a variable wavevector exponent. To see if this is also true for the wavevector dependent diffusion coefficient we have followed the same procedure and fitted the low- $k$ data with a similar functional form given as:

$$
D_{L}(k)=\frac{D_{m}}{1+\alpha|k|^{\beta}}
$$

where the coefficients $\alpha$ and $\beta$ are free fitting parameters that are not wavevector dependent. Fig. 15 shows that this functional form fits the data quite well over the range of wavevectors investigated at each packing fraction.

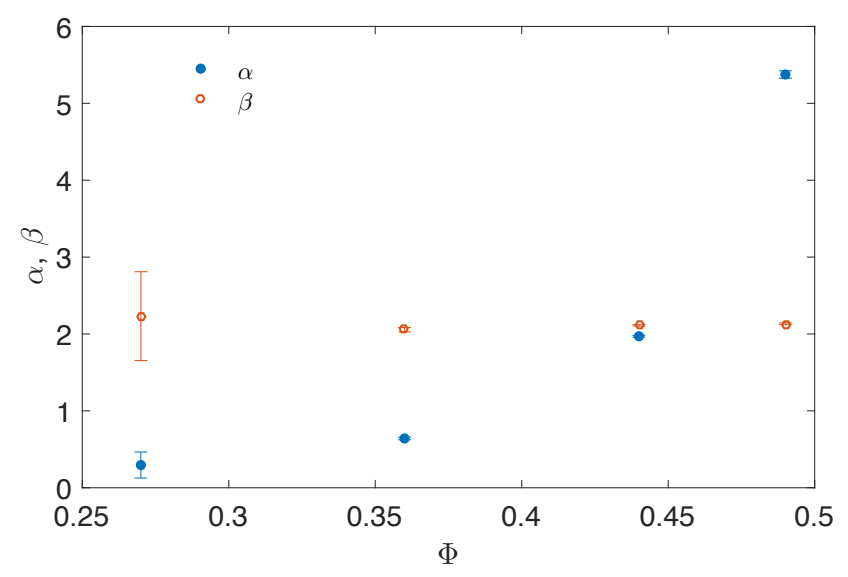

FIG. 16. Packing fraction dependence of fit coefficients $\alpha$ and $\beta$ from Eq. (27). Error bars calculated based on the standard errors of fit coefficients in Fig. 15.

The packing fraction dependence of the parameters $\alpha$ and $\beta$ are shown in Fig. 16. From this figure it is seen that the exponent $\beta$ is fairly constant over the range of packing fractions studied. Its value is very close to 2 , which indicates that the fitting function is in fact a Lorentzian. The $\alpha$ coefficient however, shows a strong dependence on the packing fraction. As the packing fraction increases towards the freezing point, $\alpha$ increases by a factor of 18 over the range studied. The increasing value of $\alpha$ reinforces the idea that the wavevector needed to achieve the macroscopic diffusive limit decreases as the packing fraction increases. This arises because as $\alpha$ increases, lower values of $k$ are needed before the term $\alpha|k|^{\beta}$ in Eq. (27) is effectively zero.

\section{CONCLUSION}

Molecular dynamics simulations were conducted on a model colloidal suspension with explicit solvent. In this study, we extended previous work and further tested the validity of the model. This was done by studying dynamical properties such as the velocity autocorrelation function, diffusion coefficients and the intermediate scat- 
tering function which were then compared with available experimental data.

The velocity autocorrelation function was found to have velocity reversals for all moderate to high packing fractions $(\Phi \geq 0.09)$. This behavior is also seen in experimental systems at $\Phi \geq 0.289$, but is only observed in single component HS systems at much higher packing fractions $(\Phi \geq 0.44)$. This indicates that inclusion of a solvent is needed in order to get dynamics which best match experimental colloidal suspensions.

The self diffusion coefficient was found to decrease as the packing fraction increased, showing that interactions with other colloidal particles inhibit motion through the solvent. This model over-predicted the value of the normalized self-diffusion coefficient, possibly due to the modified interaction parameters used to reduce depletion effects. The self diffusion coefficient extrapolates to zero near the melting point $(\Phi=0.544$ compared to $\left.\Phi_{m}=0.545\right)$ where the diffusive motion of the colloidal particles is completely inhibited and the system crystallizes.

Unlike the self diffusion coefficient, the mutual diffusion coefficient was found to increase as the packing fraction increased. This was due almost entirely to the increase in the thermodynamic factor on the approach to the freezing point. The mutual diffusion coefficient was shown to govern the low- $k$ decay of the intermediate scattering function, and so an increase in the coefficient corresponded to the increase in the decay rate of the intermediate scattering function at low wavevectors.

Lastly, after performing a multiexponetial analysis of the intermediate scattering function we found that the decay can be accurately modeled with two real exponentials (for the two diffusive decay modes) and one complex conjugate pair of exponentials (for the short time non-diffusive behavior). The two real exponential decay modes are similar to what is seen in experimental systems, and are usually associated with long-time and short-time diffusion coefficients.
Both the short-time and long-time diffusion coefficients decreased monotonically with increasing wavevector, while their corresponding mode amplitudes showed a non-monotonic dependence. The amplitude of the shorttime exponential mode decreased to zero in the $k \rightarrow 0$ macroscopic diffusive limit, indicating that this is a kinetic mode which only exists for non-zero $k$. The longtime mode remained in the low- $k$ limit, leading to a single exponential decay of the intermediate scattering function. The decay rate of this thermodynamic mode in the $k \rightarrow 0$ limit was found to be equal to the macroscopic mutual diffusion coefficient calculated independently from Green-Kubo and Kirkwood-Buff theory.

We found that the minimum wavevector needed to be effectively in the macroscopic diffusive limit (single exponential decay) decreased as the packing fraction increased, and a simple extrapolation shows the minimum wavevector going to zero at the melting point where macroscopic diffusion can no longer occur. This indicates that the packing fraction where the fluid completely crystallizes may be predicted by studying the decay of the density fluctuations of the fluid well below the melting point.

By studying the two diffusive modes we were also able to test the scaling law proposed by Segrè and Pusey. We found that the ratio of the long and short time diffusion coefficients around the structure factor peak was not constant, but had wavevector dependent behaviour, in disagreement with the proposed scaling law.

\section{ACKNOWLEDGMENTS}

Computational resources were provided by the $\mathrm{Na}$ tional Computation Infrastructure (NCI). S.D.W.H. acknowledges the Australian government for supporting this work through the Australian post-graduate award (APA). The authors would like to thank Prof. W. van Megen for helpful comments on the manuscript.
[1] U. Gasser, J. Phys.: Condens. Matter. 21, 203101 (2009).

[2] R. P. Sear, J. Phys.: Condens. Matter. 19, 033101 (2007).

[3] V. L. Colvin, MRS Bull. 637, 41 (2001).

[4] H. Imai and Y. Oaki, MRS Bull. 35, 138 (2010)

[5] V. A. Martinez, J. H. J. Thijssen, F. Zontone, W. van Megen, and G. Bryant, J. Chem. Phys. 134, 054505 (2011).

[6] P. N. Pusey and W. van Megen, Nature (London) 320, 340 (1986).

[7] W. van Megen, R. H. Ottewill, S. M. Owens, and P. N. Pusey, J. Chem. Phys. 82, 508 (1985).

[8] W. van Megen and P. N. Pusey, Phys. Rev. A 43, 5429 (1991).

[9] P. N. Segrè, O. P. Behrend and, P. N. Pusey, Phys. Rev. E 52, 5070 (1995).

[10] W. van Megen, T. C. Mortensen, S. R. Williams and, J. Müller, Phys. Rev. E 58, 6073 (1998).
[11] S. Martin, G. Bryant, and W. van Megen, Phys. Rev. E 67, 061405 (2003).

[12] B. J. Alder and T. E. Wainwright, Phys. Rev. A 1, 18 (1970).

[13] S. Ramaswamy, Adv. Phys. 50, 297 (2001).

[14] D. L. Ermak and J. A. McCammon, J. Chem. Phys. 69, 1352 (1978).

[15] J. F. Brady and J. F. Morris, J. Fluid Mech. 348, 103 (1997).

[16] J. Rotne and S. Prager, J. Chem. Phys. 50, 4831 (1969).

[17] H. Yamakawa, J. Chem. Phys. 53, 436 (1970).

[18] W. C. Soysa, B. Dünweg, and J. R. Prakash, J. Chem. Phys. 143, 064906 (2015).

[19] A. J. C. Ladd, J. Fluid Mech. 271, 285 (1994).

[20] A. J. C. Ladd, J. Fluid Mech. 271, 331 (1994).

[21] R. D. Groot and P. B. Warren, J. Chem. Phys. 107, 4423 (1997). 
[22] A. Malevanets and R. Kapral, J. Chem. Phys. 110, 8605 (1999).

[23] A. Malevanets and R. Kapral, J. Chem. Phys. 112, 7260 (2000).

[24] A. Vrabecz and G. Tóth, Mol. Phys. 104, 1843 (2006).

[25] S. D. W. Hannam, P. J. Daivis, and G. Bryant, Mol. Sim. 42, 511 (2016)

[26] P. N. Pusey, J. Phys. A: Math. Gen. 11, 119 (1978).

[27] D. Orsi, A. Fluerasu, A. Moussaid, F. Zontone, L. Cristofolini, and A. Madsen, Phys. Rev. E 85, 011402 (2012).

[28] P. N. Segrè and P. N. Pusey, Phys. Rev. Lett. 77, 771 (1996).

[29] L. B. Lurio, D. Lumma, A. R. Sandy, M. A. Borthwick, P. Falus, S. G. J. Mochrie, J. F. Pelletier, M. Sutton, L. Regan, A. Malik and G. B. Stephenson, Phys. Rev. Lett. 84, 785 (2000).

[30] D. Evans and G. Morriss, Statistical Mechanics of Nonequilibrium Liquids, 3rd ed. (Academic Press, London, 1990).

[31] J. P. Hansen and I. R. McDonald, Theory of Simple Liquids (Elsevier Science, 2006).

[32] S. R. de Groot and P. Mazur, Non-equilibrium thermodynamics (Dover Publications, New York, 1984).

[33] J. G. Kirkwood and F. P. Buff, J. Chem. Phys. 19, 774 (1951)

[34] Y. Zhou and G. H. Miller, J. Phys. Chem. 100, 5516 (1996)

[35] N. A. T. Miller, P. J. Daivis, I. K. Snook, and B. D. Todd, J. Chem. Phys. 139, 144504 (2013).

[36] Note that [35] uses an incorrect relation for the conversion of the thermodynamic factor to mass concentration units and contains some inaccurate results for $G_{\alpha \beta}$. An erratum is currently being prepared.

[37] J. W. Nichols, S. G. Moore, and D. R. Wheeler. Phys. Rev. E. 80, 051203 (2009).

[38] F. Barocchi, U. Bafile, and M. Sampoli, Phys. Rev. E 85, 022102 (2012).
[39] F. Barocchi and U. Bafile, Phys. Rev. E 87, 062133 (2013).

[40] F. Barocchi, E. Guarini, and U. Bafile, Phys. Rev. E 90, 032106 (2014).

[41] MATLAB version 8.5 (R2015a), (The MathWorks Inc., Natick, MA, 2016).

[42] S. Hess, M. Kröger, H. Voigt. Physica A. 250, 58 (1998).

[43] M. McPhie, Ph.D. thesis, RMIT University, 2003.

[44] I. Snook, B. O'Malley, M. McPhie, and P. Daivis. J. Mol. Liq. 103, 405 (2003).

[45] S. Plimpton, J. Comp. Phys., 117, 1 (1995)

[46] M. E. Tuckerman, J. Alejandre, R. López-Rendón, A. L. Jochim, and G. J. Martyna, J. Phys. A: Math. Gen. 39, 5629 (2006).

[47] S. R. Williams, G. Bryant, I. K. Snook, and W. van Megen, Phys. Rev. Lett. 96, 087801 (2006).

[48] S. R. Williams, P. McGlynn, G. Bryant, I. K. Snook, and W. van Megen, Phys. Rev. E. 74, 031204 (2006).

[49] R. Zwanzig and M. Bixon, Phys. Rev. A 2, 2005 (1970).

[50] W. van Megen, Phys. Rev. E. 73, 020503 (2006).

[51] I. Moriguchi, J. Chem. Phys. 106, 8624 (1997).

[52] W. van Megen and S. M. Underwood, J. Chem. Phys. 91, 552 (1989).

[53] A. van Blaaderen, J. Peetermans, G. Maret, and J. K. G. Dhont, J. Chem. Phys. 96, 459 (1992).

[54] A. J. Banchio, G. Nägele, and J. Bergenholtz, J. Chem. Phys. 113, 3381 (2000).

[55] W. van Megen, Phys. Rev. E. 76, 061401 (2007).

[56] B. Widom, J. Chem. Phys. 39, 2808 (1963).

[57] K. Schnell, T. J. H. Vlugt, J. M. Simon, D. Bedeaux, and S. Kjelstrup, Chem. Phys. Lett. 504, 199 (2011).

[58] J. W. Nichols and D. R. Wheeler. Ind. Eng. Chem. Res. 54, 12156 (2015).

[59] J. S. Hansen, P. J. Daivis, K. P. Travis, and B. D. Todd, Phys. Rev. E. 76, 041121 (2007). 